\title{
The chromatin remodeling subunit Baf200 promotes normal hematopoiesis and inhibits leukemogenesis
}

\author{
Lulu Liu ${ }^{1,2 \dagger}$, Xiaoling Wan ${ }^{2,3 \dagger}$, Peipei Zhou ${ }^{2,3}$, Xiaoyuan Zhou ${ }^{3,5}$, Wei Zhang ${ }^{2,7}$, Xinhui Hui ${ }^{2,7}$, Xiujie Yuann ${ }^{2,3}$, \\ Xiaodan Ding ${ }^{2,3}$, Ruihong Zhu ${ }^{2,3}$, Guangxun Meng ${ }^{2,3}$, Hui Xiao ${ }^{2,3}$, Feng Ma ${ }^{6}$, He Huang ${ }^{8}$, Xianmin Song ${ }^{9}$, \\ Bin Zhou ${ }^{4^{*}}$, Sidong Xiong ${ }^{1 *}$ and Yan Zhang ${ }^{2,3^{*}}$ (D)
}

\begin{abstract}
Background: Adenosine triphosphate (ATP)-dependent chromatin remodeling SWI/SNF-like BAF and PBAF complexes have been implicated in the regulation of stem cell function and cancers. Several subunits of BAF or PBAF, including BRG1, BAF53a, BAF45a, BAF180, and BAF250a, are known to be involved in hematopoiesis. Baf200, a subunit of PBAF complex, plays a pivotal role in heart morphogenesis and coronary artery angiogenesis. However, little is known on the importance of Baf200 in normal and malignant hematopoiesis.
\end{abstract}

Methods: Utilizing Tie2-Cre-, Vav-iCre-, and Mx1-Cre-mediated Baf200 gene deletion combined with fetal liver/bone marrow transplantation, we investigated the function of Baf200 in fetal and adult hematopoiesis. In addition, a mouse model of MLL-AF9-driven leukemogenesis was used to study the role of Baf200 in malignant hematopoiesis. We also explored the potential mechanism by using RNA-seq, RT-qPCR, cell cycle, and apoptosis assays.

Results: Tie2-Cre-mediated loss of Baf200 causes perinatal death due to defective erythropoiesis and impaired hematopoietic stem cell expansion in the fetal liver. Vav-iCre-mediated loss of Baf200 causes only mild anemia and enhanced extramedullary hematopoiesis. Fetal liver hematopoietic stem cells from Tie2-Cre ${ }^{+}, \mathrm{Baf200}^{f / f}$ or ${\mathrm{Vav}-i \mathrm{Cr}{ }^{+}, \mathrm{Baf200}}^{f / f}$ embryos and bone marrow hematopoietic stem cells from Vav-iCre ${ }^{+}, \mathrm{Baf}_{200} \mathrm{O}^{f / f}$ mice exhibited impaired long-term reconstitution potential in vivo. A cell-autonomous requirement of Baf200 for hematopoietic stem cell function was confirmed utilizing the interferon-inducible Mx1-Cre mouse strain. Transcriptomes analysis revealed that expression of several erythropoiesis- and hematopoiesis-associated genes were regulated by Baf200. In addition, loss of Baf200 in a mouse model of MLL-AF9-driven leukemogenesis accelerates the tumor burden and shortens the host survival.

Conclusion: Our current studies uncover critical roles of Baf200 in both normal and malignant hematopoiesis and provide a potential therapeutic target for suppressing the progression of leukemia without interfering with normal hematopoiesis.

Keywords: Hematopoiesis, Leukemogenesis, Chromatin remodeling complex, Epigenetics

\footnotetext{
*Correspondence: zhoubin@sibs.ac.cn; sdxiong@suda.edu.cn;

yan_zhang@sibs.ac.cn

${ }^{\dagger}$ Equal contributors

${ }^{4}$ The State Key Laboratory of Cell Biology, CAS Center for Excellence in Molecular Cell Science, Shanghai Institute of Biochemistry and Cell Biology,

Chinese Academy of Sciences, 320 Yue-Yang Road, Shanghai, China

${ }^{1}$ Institute of Biology and Medical Sciences, Soochow University, No. 199 Ren'ai Rd, Suzhou, China

${ }^{2}$ Key Laboratory of Molecular Virology and Immunology, Institut Pasteur of

Shanghai, Chinese Academy of Sciences, 320 Yue-Yang Road, Shanghai,

China

Full list of author information is available at the end of the article
} 


\section{Background}

Hematopoiesis is a continuous process with a rare population of hematopoietic stem cells (HSCs) giving rise to all blood cell types. It is finely orchestrated by both cellintrinsic factors and microenvironmental clues [1]. In mammals, hematopoiesis occurs sequentially during the development in the yolk sac (YS), aorta-gonadmesonephros (AGM) region, placenta, fetal liver (FL), and bone marrow (BM). After birth, hematopoiesis continues with balanced proliferation, differentiation, quiescence, and apoptosis of HSCs. Various transcription factors, signaling pathways, and epigenetic regulators are involved in the regulation of these processes, and abnormalities occurring in these processes cause hematopoietic disorders including anemia or malignant transformation [2-11]. Therefore, defining factors involved in the regulation of hematopoiesis is an important issue in HSC biology.

Adenosine triphosphate (ATP)-dependent chromatin remodeling complexes include five different classes, SWI/ SNF, ISWI, NuRD/Mi2/CHD, INO80, and SWR1, and are evolutionarily conserved. The SWI/SNF complexes of mammals include the BRG1-associated factor (BAF) complex and polybromo BRG1-associated factor (PBAF) complex. These two complexes consist of several common subunits and specific subunits. The common subunits include BRG1, BAF170, BAF155, BAF60a/b/c, BAF57, $\mathrm{BAF} 53 \mathrm{a} / \mathrm{b}, \mathrm{BAF} 47, \mathrm{BAF} 45 \mathrm{a} / \mathrm{b} / \mathrm{c} / \mathrm{d}$, and actin. BRM and BAF250a/b subunits are found only in the BAF complex; BAF200, BAF180, and BRD7 subunits are limited to the PBAF complex [12]. BRG1, BAF53a, BAF45a, BAF180, and BAF250a are known to be involved in hematopoiesis [13-22], but the hematopoietic activity of other BAF or PBAF subunits has not been determined.

BAF200, also known as ARID2, encoded by the BAF200 gene, is a unique subunit of the PBAF chromatin remodeling complex, and inactivating mutations have been reported in a variety of human cancers [23-25]. Baf200-null mutant mice displayed embryonic lethality with multiple cardiac defects [26]. A recent study revealed a correlation between BAF200 defect and myelodysplasia [27]. However, the precise role of Baf200 in hematopoiesis has not been examined so far. Here, we investigated the function of Baf200 in hematopoiesis through conditional deletion approach using the Tie2-Cre, Vav-iCre, and Mx1-Cre mice. Tie2-Cre-mediated loss of Baf200 causes embryonic lethality with defective fetal erythropoiesis, a reduced HSC pool in the FL, and impaired long-term reconstitution capacity of FL HSCs. Vav-iCre-mediated loss of Baf200 causes mild anemia and increased extramedullary hematopoiesis in adult mice. The long-term reconstitution potentials of the FL or BM HSCs from $\mathrm{Vav}-i \mathrm{Cre}{ }^{+}, \mathrm{Baf}_{200^{f / f}}$ mice are severely impaired. The loss of Baf200 alters the transcription of a cohort of genes involved in the maintenance of HSC homeostasis. In addition, Baf200 deficiency accelerates the progression of MLL-AF9-induced leukemia. Taken together, the results demonstrate the involvement of Baf200 in both normal and malignant hematopoiesis and provide additional knowledge of the cellular and genetic activity of the chromatin remodeling complex in HSC function.

\section{Methods}

Mice

The Baf200 ${ }^{\mathrm{LacZ} /+}$ mice line was described previously [26]. Baf200 ${ }^{\mathrm{LacZ} /+}$ mice were crossed with Actin-Flpe transgenic mice to generate $B a f 200^{f /+}$ mice. Then, Baf200 $0^{f /+}$ mice were further crossed with heterozygous Tie2-Cre, Vav-iCre, or Mx1-Cre transgenic mice to generate Tie2-Cre ${ }^{+}, \mathrm{Baf}_{200^{f / f}}$, Vav-iCre ${ }^{+}, \mathrm{Baf}_{200^{f / f}}$, or Mx1-Cre ${ }^{+}$, $B a f 200^{f / f}$ mice. All mice were bred under specific pathogen-free conditions. The protocols were approved by the Institutional Animal Care and Use Committee (IACUC) in Institut Pasteur of Shanghai. Genotyping and gene deletion efficiency were performed by polymerase chain reaction (PCR) using primers specific for wild-type (WT) Baf200 alleles, floxed exon4 or deleted exon4. Gene deletion efficiency was also determined by reverse transcription-quantitative PCR (RT-qPCR) using primers in exon3 and exon4 (see Additional file 1: Table S2 and Table S3 for the primers).

\section{Flow cytometry}

FL, BM, spleen, and thymus cells were isolated and passed through a 40- $\mu \mathrm{m}$ nylon cell strainer (BD Biosciences) and stained for $20 \mathrm{~min}$ on ice in PBS supplemented with $2 \%$ FBS. Dead cells were discarded from analysis by 4,6-diamino-2-phenylindole (DAPI) (Molecular Probes). All the antibodies used in the experiments are summarized in Additional file 1: Table S4. Flow cytometric analysis was performed on LSRII or Fortessa (BD Biosciences), and flow sorting was performed on FACSAriaII (BD Biosciences). Data were analyzed by FlowJo software (Tree Star, Ashland, OR).

\section{FL cell counting}

Embryos were collected from female mice at days 12.5 to 17.5 of pregnancy, and the FLs dissected from each embryo were removed into $1 \mathrm{~mL}$ PBS supplemented with $2 \%$ FBS. To obtain single cells, the FLs were pipetted by $1 \mathrm{~mL}$ pipette gently and passed through a $40-\mu \mathrm{m}$ nylon cell strainer (BD Biosciences). Then, the cell number was counted by hemocytometer.

\section{Transplantation assay}

For competitive FL transplantation assay, E14.5 WT control and Tie2-Cre ${ }^{+}, \mathrm{Baf}_{200^{f / f}}$ or $\mathrm{Vav}-i \mathrm{Cre} e^{+}, \mathrm{Baf}_{200^{f / f}} \mathrm{FL}$ donor cells $\left(\mathrm{CD} 45.2^{+}\right)$were isolated from timed pregnancies and single-cell suspensions were made using $40-\mu$ m nylon cell 
strainers (BD Biosciences). $2 \times 10^{6}$ donor cells (CD45.2 ${ }^{+}$) were mixed with $1 \times 10^{6} \mathrm{C} 57 \mathrm{BL} / 6 \mathrm{BM}$ competitor cells $\left(\mathrm{CD} 45.1^{+}\right)$prior to injection into lethally irradiated $(9.5 \mathrm{~Gy}$, $\mathrm{X}$-ray) $\mathrm{CD} 45.1^{+} \mathrm{CD} 45.2^{+} \mathrm{C} 57 \mathrm{BL} / 6$ recipient mice. For competitive BM transplantation assay, $1 \times 10^{6} \mathrm{WT}$ or $\mathrm{Vav}-\mathrm{iCre}^{+}$, Baf200 ${ }^{f / f} \mathrm{BM}$ donor cells $\left(\mathrm{CD} 45.2^{+}\right)$were mixed with $1 \times 10^{6} \mathrm{C} 57 \mathrm{BL} / 6 \mathrm{BM}$ competitor cells $\left(\mathrm{CD} 45.1^{+}\right)$prior to injection into lethally irradiated (9.5 Gy, X-ray) CD45.1 ${ }^{+} \mathrm{CD} 45.2^{+} \mathrm{C} 57 \mathrm{BL} / 6$ recipient mice. Sixteen weeks later, the hematopoietic tissues were harvested and stained with antibodies for flow cytometry analysis to determine the ratios of donor- versus competitor-derived cells. Under the Mx1-Cre condition, $2 \times 10^{6}$ WT or Mx1-Cre ${ }^{+}$, Baf $200^{f / f}$ donor cells (CD45.2 $2^{+} \mathrm{BM}$ or FL) were mixed with $1 \times 10^{6}$ C57BL/6 BM competitor cells $\left(\mathrm{CD} 45.1^{+}\right)$prior to injection into lethally irradiated (9.5 Gy, X-ray) $\mathrm{CD} 45.1^{+} \mathrm{CD} 45.2^{+}$ C57BL/6 recipient mice, the recipient mice were bled 6-8 weeks after transplantation to analyze the percentage of chimerism in their blood and then injected with poly(I:C) (GE Healthcare Life Science) to induce the deletion of Baf200 gene, and next, the reconstitution ability of donor cells was evaluated in the peripheral blood of recipient 1,2 , and 3 months after pIpC injection. For the assessment of the niche effect in hematopoiesis, $2 \times 10^{6}$ control CD45.1 $1^{+} \mathrm{BM}$ cells were transplanted into

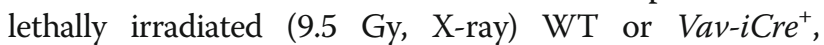
Baf200 $0^{f / f}$ mice. Three months later, $6 \times 10^{6} \mathrm{BM}$ cells from recipient in the first transplant were mixed with $1 \times 10^{6}$ CD 45. $1^{+} \mathrm{CD} 45.2^{+} \mathrm{BM}$ cells prior to injection into lethally irradiated (9.5 Gy, X-ray) CD $45.2^{+}$C57BL/6 recipient mice. For homing assay, $2 \times 10^{7} \mathrm{FL}$ cells from E14.5 WT or Tie2-Cre ${ }^{+}$, Baf200 2 fff embryos or $2 \times 10^{7} \mathrm{BM}$ cells from WT or $\mathrm{Vav}^{-i C r e}{ }^{+}$, Baf200 fff mice labeled with $2 \mu \mathrm{M}$ CellTrace Violet (Invitrogen) were transplanted into lethally irradiated $\mathrm{CD} 45.2^{+} \mathrm{C} 57 \mathrm{BL} / 6$ recipient mice. Percentage of donor cells in the recipients was detected $16 \mathrm{~h}$ post-transplantation.

\section{Cell cycle and apoptosis analysis}

For FL cell cycle analysis, pregnant female mice were injected intraperitoneally with BrdU for $1 \mathrm{~h}$, and for BM LSK cell cycle analysis, WT or $\mathrm{Vav}-i \mathrm{Cre}^{+}, \mathrm{Baf} 20 \mathrm{f}^{f / f}$ mice were injected intraperitoneally with BrdU for $2 \mathrm{~h}$. Then, FL or BM cells were isolated and stained with antibodies, followed by fixation, permeabilization, and stained with anti-BrdU antibody and Hoechst 33342 (Molecular Probes) according to the manufacturer's instruction (BD Biosciences). For Ki67 staining, FL or BM cells were stained with surface markers to define subsets, followed by fixation, permeabilization, and stained with anti-Ki67 antibody and Hoechst 33342.

To detect apoptotic cells, FL or BM cells were stained with surface markers to define subsets, followed by staining with DAPI and anti-annexin V antibody in accordance with the manufacturer's instruction (BD Biosciences).

\section{Histology}

The FL, spleen, liver, and bone (femur and tibia) tissues were fixed in $4 \%$ paraformaldehyde overnight at room temperature. To decalcify, the bone tissues were placed into 10\% EDTA solution and the solution was changed every other day for about 2 weeks. Then, the tissues were embedded in paraffin, sectioned at $6 \mu \mathrm{m}$, and stained with hematoxylin-eosin.

\section{Retroviral production, transformation, and leukemia mouse model}

Retroviral supernatants were produced using HEK293T cells as described previously [28]. For leukemia cell production, $\mathrm{Lin}^{-} \mathrm{BM}$ cells were isolated, pre-stimulated overnight, and infected with retrovirus. Infected cells were injected into lethally (9.5 Gy, X-ray) irradiated recipients. Leukemia cells were harvested from the sick mice and transplanted into sub-lethally (5 Gy, X-ray) irradiated recipients in secondary transplantation.

\section{RNA-seq and RT-qPCR}

Total RNA was isolated from flow-sorted LSK cells or S3 cells using the RNeasy Mini kit (QIAGEN, Valencia, CA), and the RNA-seq analysis was performed at BGI (The Beijing Genomics Institute) via Illumina HiSeq ${ }^{\mathrm{m}}$ 2000. Briefly, the mRNA was enriched by using the oligo (dT) magnetic beads, then fragmented into fragments. Then, the double-stranded complementary DNA (cDNA) was synthesized and purified by magnetic beads. End reparation and 3 '-end single nucleotide A (adenine) addition was then preformed. Finally, sequencing adaptors were ligated to the fragments. The fragments were enriched by PCR amplification, and the quality of the library was assessed by using Agilent 2100 Bioanalyzer and ABI StepOnePlus Real-Time PCR system. Then, the library was ready for sequencing. Raw data were deposited in the Sequence Read Archive database (accession number SRP117796). Functional Gene Ontology analysis of differential genes was determined by using DAVID Bioinformatics Resources 6.8 [29, 30].

For RT-qPCR analysis of gene expression in LSK cells, cDNA was produced by EZ-press Cell to cDNA Kit (EZbioscience). For RT-qPCR analysis of gene expression in other cells, total RNA was isolated by TRIzol (invitrogen) and converted into cDNA by using FastKing RT Kit (Tiangen). Primers for PCR were designed using Primer3 software or selected from PrimerBank database (Additional file 1: Table S3). All the reactions were performed at ABI Prism 7900HT (Applied Biosystems) using SYBR $^{\oplus}$ Premix Ex Taq ${ }^{\mathrm{Tm}}$ II (Takara). 


\section{Statistical analysis}

The significance of differences was determined with twotailed paired Student's $t$ tests by using Prism software (GraphPad Software). Survival data from leukemia mouse models were analyzed by using a log-rank nonparametric test, and the data were expressed as Kaplan-Meier survival curves. Statistical significance is indicated by ${ }^{*} p<0.05,{ }^{* *} p$ $<0.01$, or ${ }^{* * * *} p<0.001$.

\section{Results}

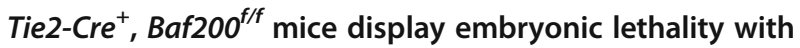
defective fetal erythropoiesis

The expression of Baf200 was examined in representative hematopoietic lineages. Baf200 is widely expressed in all hematopoietic lineages and particularly in FL LSK (Lin $\left.{ }^{-} \mathrm{Scal}^{+} \mathrm{c}-\mathrm{Kit}^{+}\right)$, FL hematopoietic progenitor cell (HPC) $\left(\mathrm{Lin}^{-} \mathrm{Scal}^{-} \mathrm{C}-\mathrm{Kit}^{+}\right), \quad$ FL $\quad$ S2 $\quad\left(\right.$ Ter119 $\left.{ }^{\mathrm{low}} \mathrm{CD} 71^{+}\right), \quad$ FL $\quad$ S3 (Ter119 ${ }^{\text {high }} \mathrm{CD} 71^{+}$), adult BM short-term (ST)-HSC (Lin ${ }^{-} \mathrm{Sca} 1^{+} \mathrm{C}_{-} \mathrm{Kit}^{+} \mathrm{CD} 34^{+} \mathrm{Flt} 3^{\text {low }}$ ), multipotent progenitor (MPP) $\left(\mathrm{Lin}^{-} \mathrm{Scal}^{+}{ }^{+}-\mathrm{Kit}^{+} \mathrm{CD} 34^{+} \mathrm{Flt}^{+}{ }^{+}\right.$), megakaryocyte-erythroid progenitor (MEP) ( $\mathrm{Lin}^{-} \mathrm{Sca}{ }^{-} \mathrm{c}-\mathrm{Kit}^{+} \mathrm{CD} 34^{-} \mathrm{CD} 16 / 32^{\text {low }}$ ), and granulocyte-macrophage progenitor (GMP) $\left(\mathrm{Lin}^{-} \mathrm{Sca} 1\right.$ ${ }^{-} \mathrm{c}-\mathrm{Kit}^{+} \mathrm{CD} 34^{+} \mathrm{CD} 16 / 32^{\text {high }}$ ) lineages (Additional file 1: Figure S1). To investigate its role in hematopoiesis in vivo, Baf200 was conditionally deleted by crossing Baf200 f/f mice with Tie2-Cre transgenic mice which express Cre-recombinase in endothelial cells and hematopoietic lineages [31]. Only four Tie2-Cre ${ }^{+}$, Baf200 $0^{f f f}$ mice were obtained among 239 offspring from intercross between male Tie2$\mathrm{Cre}^{+}, \mathrm{Baf} 20 \mathrm{f}^{\mathrm{f} / \mathrm{t}}$ mice and female Baf200 $\mathrm{f}^{f / f}$ mice at weaning age, which was considerably lower than the expected Mendelian ratio (Additional file 1: Figure S2a). The four Tie2-Cre ${ }^{+}$, Baf20 f/f mice exhibited low recombination efficiency of the LoxP sites in hematopoietic cells (data not shown). Examination of the genotypes in the litters from timed mating intercross found that $\mathrm{Tie}^{2}-\mathrm{Cre}^{+}, \mathrm{Baf} 200^{\mathrm{f} f}$ embryos were alive and were recovered at the expected Mendelian ratio at embryonic day (E) 17.5, but that the majority died by E18.5 (Fig. 1a and Additional file 1: Figure S2a).

Genomic PCR or RT-qPCR confirmed the deletion of Baf200 in the FL cells of E14.5 Tie2-Cre ${ }^{+}$, Baf200 f/f embryos (Additional file 1: Figure S2c, d), suggesting that Baf200 was efficiently deleted in the hematopoietic lineages. Tie2-Cre ${ }^{+}, \mathrm{Baf}_{20} \mathrm{f}^{f / f}$ embryos showed severe anemia and profound growth retardation as early as E15.5 compared with their WT Tie2-Cre ${ }^{-}, \mathrm{Baf200^{f/f }}$ or Tie2-Cre ${ }^{-}$, Baf200 $0^{f /+}$ littermates (Fig. 1a and Additional file 1: Figure S2b). The absolute cell number of FL from Tie2-Cre ${ }^{+}$, Baf200 f/f embryos was also reduced since E13.5 (Fig. 1b). The apoptosis of FL cells in $\mathrm{Tie}_{2}-\mathrm{Cre}^{+}$, Baf200 $0^{f f f}$ and WT embryos at E14.5 and E16.5 was measured using annexin- $\mathrm{V}$ and DAPI staining followed by flow cytometric analysis. Although the apoptosis of FL cells were comparable between Tie2-Cre $e^{+}$Baf200 $0^{f / f}$ and WT embryos at E14.5, the percentage of annexin- $\mathrm{V}$ ${ }^{+}, \mathrm{DAPI}^{-}$and annexin- $\mathrm{V}^{+}, \mathrm{DAPI}^{+} \mathrm{FL}$ cells in $\mathrm{Tie} 2-\mathrm{Cre}{ }^{+}$, Baf200 f/f embryos at E16.5 is significantly higher than that in WT embryos (Additional file 1: Figure S2e). These results suggested that increased apoptosis might account, at least in part, for the reduced FL cell number in Tie2-Cre ${ }^{+}$, Baf200 fff embryos.

Evaluation of erythropoiesis in the FL from WT and Tie2-Cre ${ }^{+}$, Baf200 f/f embryos by staining with Ter119 and CD71 surface markers revealed that the percentages of S0, S1, and S2 cells were increased and the percentages of S4 and S5 cells were decreased in E15.5 Tie2-Cre ${ }^{+}$, Baf $200^{f / f} \mathrm{FL}$ (Fig. 1c, d). Although the total number of FL cells in the Tie2-Cre $e^{+}$, Baf $200^{f / f}$ embryos was reduced, the absolute numbers of S0, S1, and S2 cells remained unchanged, whereas the absolute numbers of S3, S4, and S5 cells were reduced (Fig. 1e). Notably, similar phenotype was also observed at E13.5 and E14.5 (Additional file 1: Figure S2f-g) and became much more apparent at E16.5 (Fig. 1f-h). Though the percentage of FL MEP (Lin ${ }^{-}$Sca-1 ${ }^{-} \mathrm{C}-\mathrm{Kit}^{+} \mathrm{CD} 16 / 32^{-} \mathrm{CD}^{-} 1^{-}$) was increased, the absolute cell number remained unchanged in E14.5 Tie2-Cre $e^{+}$, Baf200 ${ }^{f / f}$ FL (Additional file 1: Figure S2 h). We found that the cell cycle status and apoptosis of FL erythrocytes from Tie2-Cre ${ }^{+}$, Baf200 fff embryos were comparable to those from WT embryos (Additional file 1: Figure S2i-k), suggesting that the defective erythropoiesis in Tie2-Cre $e^{+}$, Baf200 $0^{f f f} \mathrm{FL}$ was not caused by reduced cell proliferation or increased apoptosis. These results indicate that Baf200 plays a pivotal role in the erythroid terminal differentiation in the FL.

To determine the potential mechanism by which Baf200 regulates fetal erythropoiesis, we performed RNA-seq analysis of the transcriptomes of S3 cells from E14.5 WT or Tie2-Cre ${ }^{+}$, Baf200 $0^{f / f}$ embryos. A total 161 upregulated genes and 60 downregulated genes were observed in Tie2-Cre ${ }^{+}$, Baf200 ${ }^{f / f}$ S3 cells (Fig. 1i). Functional Gene Ontology (GO) analysis of differentially expressed genes indicated significant enrichment in translation, proteolysis involved in cellular protein catabolic process, glycosphingolipid metabolic process, erythrocyte homeostasis, intracellular signal transduction, response to oxidative stress, and oxidation-reduction process terms (Additional file 1: Figure S3). During the transition from S2 to S3, the expression level of $M y b$ and Kit is downregulated [32], and the downregulation of $M y b$ is important for erythroid terminal differentiation [33, 34]. In addition, Hmox 1 overexpression in erythroid cells decreases hemoglobin synthesis [35]. Moreover, the expression of Pla2g16 is induced during erythroid maturation, and Pla2g16 deficiency leads to impaired proerythroblasts 
a

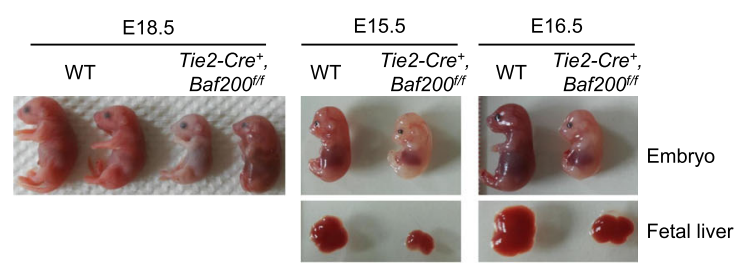

C

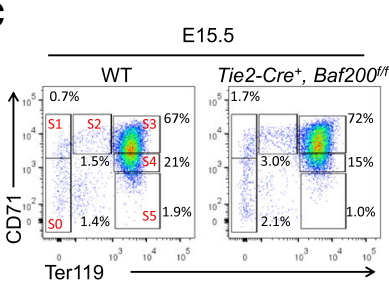

f

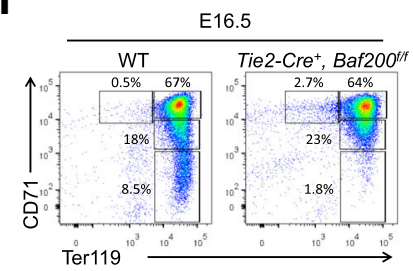

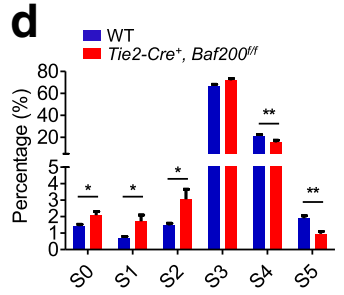

g

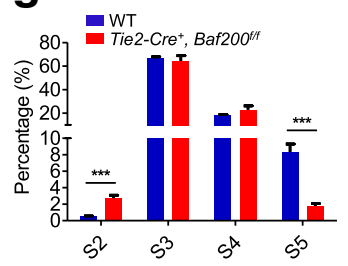

b

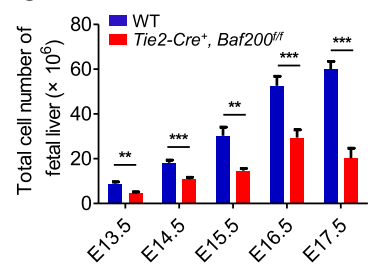

e

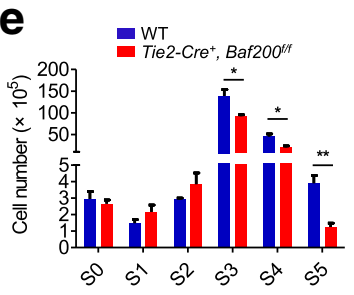

h

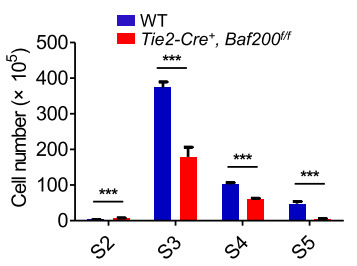

i
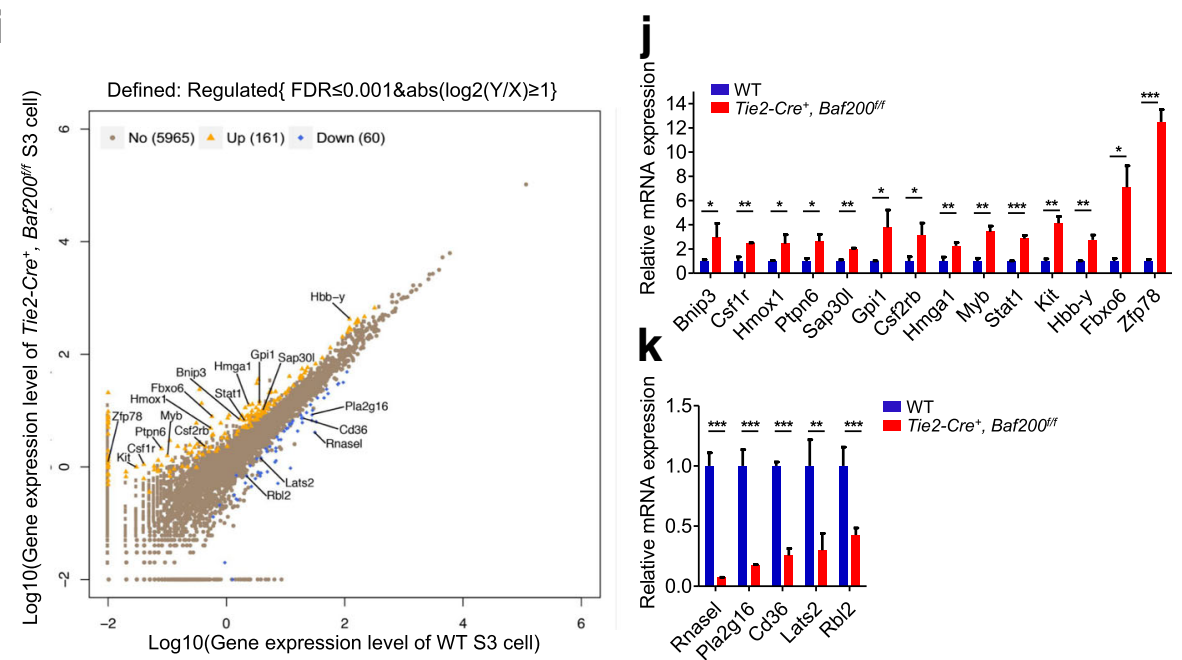

Fig. 1 Tie2-Cre ${ }^{+}$, Baf200 fff mice display embryonic lethality with defective fetal erythropoiesis. a Representative photographs of embryos and FLs from Tie2-Cre ${ }^{+}$, Baf20 f/f mice and WT littermate at E15.5, E16.5, and E18.5. b Absolute cell number of FLs from Tie2-Cre ${ }^{+}$, Baf200 $^{f / f}$ embryos and WT littermate at different developmental stages ( $n=5-13$ per genotype for each stages). c FACS profiles of CD71/Ter119 erythroid subsets in the FLs from E15.5 Tie2-Cre ${ }^{+}$, Baf200 fff embryos and WT littermates. d, e Percentage (d) and absolute cell number (e) of CD71/Ter119 erythroid subsets from E15.5 Tie2-Cre ${ }^{+}$, Baf20 fff embryos and WT littermates ( $n=4$ per genotype). $\mathbf{f}$ FACS profiles of CD71/Ter119 erythroid subsets in the FLs from E16.5 Tie2-Cre+, Baf200 f/f embryos and WT littermates. g, h Percentage (g) and absolute cell number (h) of CD71/Ter119 erythroid subsets from E16.5 Tie2-Cre ${ }^{+}$, Baf200 fff embryos and WT littermates ( $n=4$ per genotype). i Scatter plots of all expressed genes in S3 cells from WT and Tie2-Cre ${ }^{+}$, $B a f 200^{f / f} \mathrm{FLS}$. Blue indicates downregulation gene, yellow indicates upregulation gene, and gray indicates non-regulation gene in Tie2-Cre ${ }^{+}$, Baf200 ${ }^{f / f}$ S3 cells. $\mathbf{j}, \mathbf{k}$ Upregulation genes (j) and downregulation genes (k) in Tie2-Cre ${ }^{+},{\text {Baf2 } 200^{f / f}} \mathrm{~S} 3$ cells were confirmed by RT-qPCR $(n=3$ per genotype). Data are shown as means \pm SEM. ${ }^{*} P<0.05 ;{ }^{* *} P<0.01$; ${ }^{* * *} P<0.001$

(ProE) terminal differentiation [36]. We found that the expression of $M y b$, Hmox 1 , and Kit was increased, while the expression Pla2g16 was decreased in Tie2-Cre ${ }^{+}$, Baf200 fff
S3 cells (Fig. 1i-k).These may partially explain the impaired FL erythropoiesis in Tie2-Cre $e^{+}$Baf200 $0^{f / f}$ embryos. 
The expansion of FL HSCs in Tie2-Cre ${ }^{+}, \mathrm{Baf200}^{\mathrm{f} / f}$ embryos is severely impaired

We then examined the hematopoietic stem and progenitor cells in WT or Tie2-Cre ${ }^{+}$, Baf200 ${ }^{f / f}$ FL (Fig. 2a). The absolute number of LSK $\left(\mathrm{Lin}^{-} \mathrm{Scal}^{+} \mathrm{c}-\mathrm{Kit}^{+}\right)$cells and longterm (LT)-HSCs (CD150 $\left.{ }^{+} \mathrm{CD} 48^{-} \mathrm{LSK}\right)$ from $\mathrm{Tie} 2-\mathrm{Cr} e^{+}$, Baf200 $^{f f f} \mathrm{FL}$ was similar to those from WT FL at E12.5 (Fig. 2b, c). However, the absolute number of LSK cells and LT-HSC was significantly decreased in E13.5 and

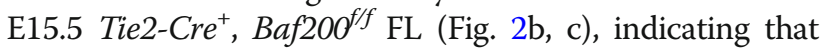
the expansion, but not the initial seeding, of HSCs in the FL was impaired in Tie2-Cre ${ }^{+}$, Baf200 fff embryos. We next detected the cell proliferation and apoptosis of LSK and CD150 ${ }^{+}$LSK cells from E14.5 Tie2-Cre ${ }^{+}$, Baf200 fff embryos. There was a significant increase in apoptosis of the LSK and $\mathrm{CD} 150^{+}$LSK cells of Tie2-Cre ${ }^{+}$, Baf200 $0^{f / f}$ embryos, while the cell proliferation remains unchanged (Fig. 2d, e). Hence, Tie2-Cre-mediated loss of Baf200 causes aberrant apoptosis of FL LSK and LT-HSC.

To further identify the downstream mediators of Baf200 in FL HSCs, we analyzed the transcriptomes of FL LSK cells from E14.5 WT or Tie2-Cre ${ }^{+}, \mathrm{Baf}_{200^{f / f}} \mathrm{em}-$ bryos by RNA-seq. A total of 94 genes were upregulated and 77 genes were downregulated in Tie2-Cre, Baf200 f/f FL LSK cells compared with WT cells (Additional file 1: Figure S4a). GO analysis of these 171 differentially expressed genes indicated significant enrichment in cell adhesion, reactive oxygen species metabolic process, inflammatory response, and phagocytosis pathway, as well as hemopoiesis (Additional file 1: Figure S4c). Alterations in the expression of hematopoiesisassociated genes, including Hck, Csf1r, Procr, Gprasp2, Fstl1, and Tbxas1 [37-41], were confirmed by RT-qPCR (Additional file 1: Figure S4b).

\section{Baf200 is required for the FL HSC maintenance}

A competitive repopulation assay was performed by cotransplanting FL cells from CD45.2 $2^{+}$Tie2-Cre ${ }^{+}, \mathrm{Baf}_{2} 00^{f / f}$ embryos or CD $45.2^{+}$WT controls with $\mathrm{CD} 45.1^{+} \mathrm{BM}$ cells at a ratio of 2:1 into lethally irradiated CD45.1 ${ }^{+} \mathrm{CD} 45.2^{+}$recipient mice (Fig. 3a). Reconstitution by donor cells was evaluated in the peripheral blood, spleen, thymus, and BM of the recipient mice 16 weeks post-transplantation. As expected, WT donor cells competed effectively with the competitor cells. In contrast, Tie2-Cre ${ }^{+}, \mathrm{Baf} 200^{f / f} \mathrm{FL}$ cells failed to contribute the long-term reconstitution of the myeloid, $\mathrm{T}$, and $\mathrm{B}$ cell lineages or the LSK cells in the chimeras (Fig. 3b and Additional file 1: Figure S5a, b). The homing capacity of Tie2-Cre ${ }^{+}$, Baf20 fif FL cells remains unchanged (Additional file 1: Figure S5c). We next generated an inducible Baf200-deletion mouse model by crossing Mx1-Cre with Baf200-flox mice. FL cells from Mx1-Cre ${ }^{+}$, Baf200 flf (before deletion) or control embryos were transplanted together with competitor BM cells into lethally irradiated recipients. Six to 8 weeks later, the recipient mice received poly(I:C), and the peripheral blood was analyzed for 3 months (Fig. 3c). Deletion of a
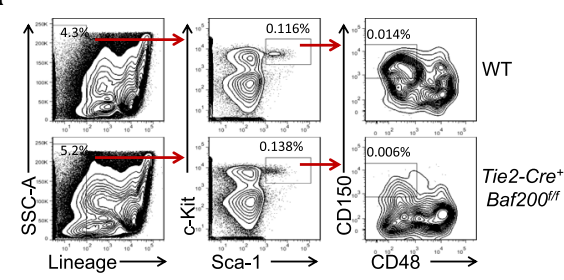
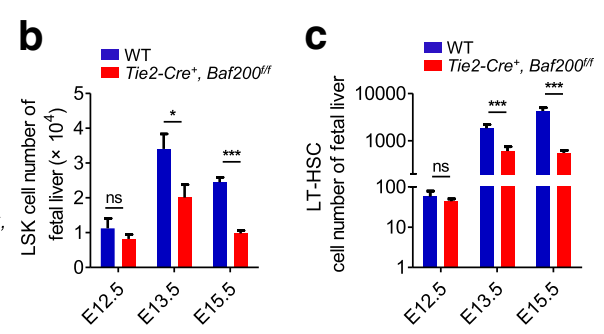

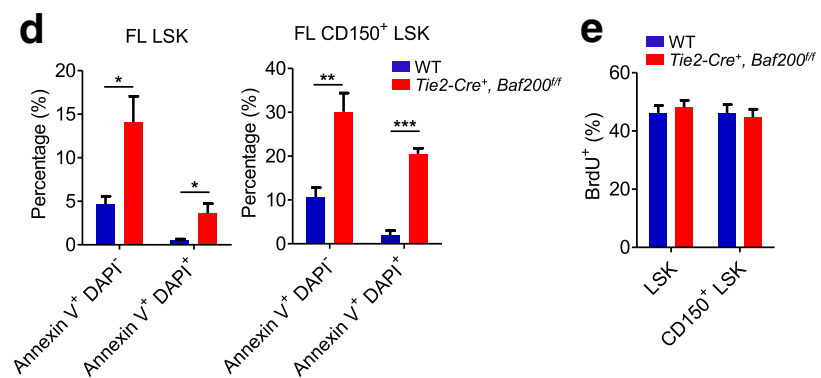

Fig. 2 The expansion of FL HSCs in Tie2-Cre ${ }^{+}$, Baf200 f/f embryos is severely impaired. a FACS profiles of LSK and LT-HSC (CD150+CD48 ${ }^{-}$LSK) subsets in the FLs from E15.5 Tie2-Cre ${ }^{+}$, Baf200 fff embryos and WT littermates. b, c Absolute cell number of LSK cells (b) and LT-HSCs (c) from Tie2-Cre ${ }^{+}$, Baf200 fff embryos and WT littermates at indicated stages. $\mathbf{d}$ Percentage of apoptotic cells of LSK and CD150 LSK cells from E14.5 Tie2-Cre ${ }^{+}$, Baf200 fff embryos $(n=4)$ and WT littermates $(n=5)$. The apoptosis was evaluated by using annexin V and DAPI. e Cell cycle status of LSK and CD150 ${ }^{+}$LSK cells from E14.5 Tie2-Cre ${ }^{+}$, Baf200 ${ }^{f / f}$ embryos $(n=4)$ and WT littermates $(n=5)$. No difference of BrdU ${ }^{+}$cells was observed. Data are shown as means \pm SEM. ${ }^{*} P<0.05 ;{ }^{* *} P<0.01 ;{ }^{* *} P<0.001$. ns indicates no significant difference 


\section{a}

Donor: CD45.2+ FL cells from WT or Tie2-Cre ${ }^{+}$, Baf200 ${ }^{\text {fff }}$ mice

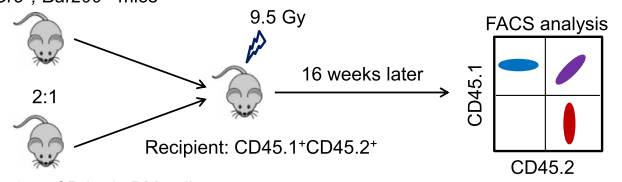

Competitor: CD45.1+ BM cells

b
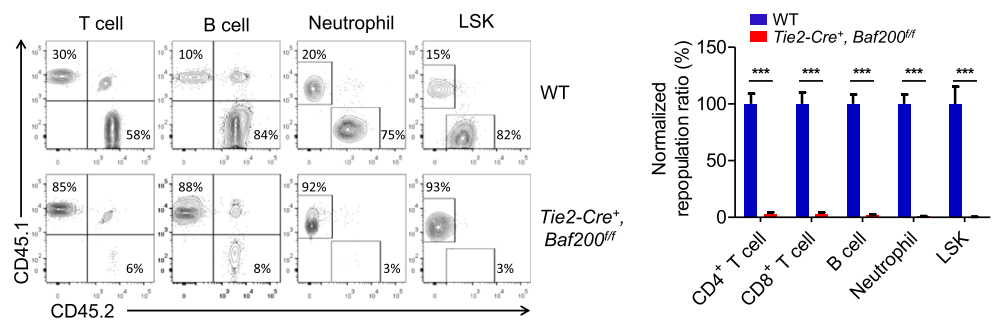

C

Donor: CD45.2+ FL cells from WT or $\mathrm{Mx} 1-\mathrm{Cre}^{+}, \mathrm{Baf200^{ff }}$ mice

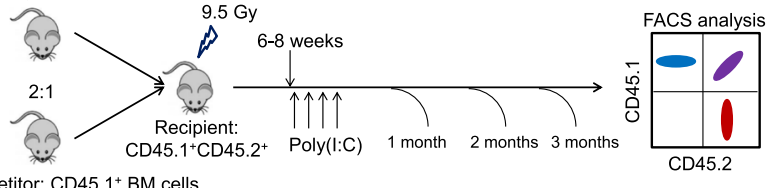

Competitor: $\mathrm{CD} 45.1^{+} \mathrm{BM}$ cells
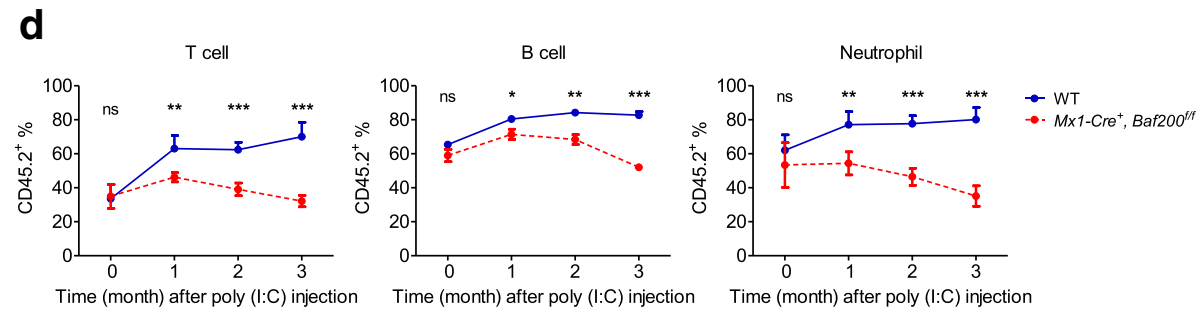

Fig. 3 Baf200 is required for the FL HSC maintenance. a Scheme of competitive FL transplantation assay. b Representative FACS profiles showing the frequency of T cells $\left(\mathrm{CD}^{+}\right), \mathrm{B}$ cells $\left(\mathrm{B} 22 \mathrm{O}^{+}\right)$, neutrophils $\left(\mathrm{Gr} 1^{\text {high }} \mathrm{CD} 11 \mathrm{~b}^{+}\right)$in the peripheral blood, and $\mathrm{LSK}\left(\mathrm{Lin}^{-} \mathrm{Sca}{ }^{+} \mathrm{C}-\mathrm{Kit}^{+}\right)$cells in the BM from recipient mice 16 weeks after transplantation (left) and the graph showing the relative ratios of CD45.2 versus CD45.1 of the indicated cell types in recipient mice 16 weeks after transplantation (right) ( $n=5$ per genotype). c Scheme of competitive FL transplantation assay in Mx1-Cre mouse strain. $\mathbf{d}$ Percentage of donor-derived T cells (left), B cells (middle), and neutrophils (right) in the peripheral blood from recipient mice assessed at different time points ( $n=5$ per genotype for each subset). Data are shown as means \pm SEM. ${ }^{*} P<0.05 ;{ }^{* *} P<0.01 ;{ }^{* * *} P<0.001$

Baf200 led to gradual but significant decrease of lymphoid and myeloid lineages in the recipients (Fig. 3d). These results suggest that a cell-intrinsic role of Baf200 is required for FL HSC function.

\section{Baf200 contributes to adult erythropoiesis}

To further explore the in vivo function of Baf200 in hematopoiesis, we generated $\mathrm{Vav}-i \mathrm{Cre}^{+}, \mathrm{Baf} 200^{f / f}$ mice by utilizing Vav-iCre mice strain which express Cre-recombinase in hematopoietic lineages [42]. The $\mathrm{Vav}_{-i \mathrm{Cr}}{ }^{+}, \mathrm{Baf} 200^{f / f}$ mice were recovered at the expected Mendelian ratio and displayed an appearance indistinguishable from the $\mathrm{Vav}-\mathrm{iCre} \mathrm{C}^{-}, \mathrm{Baf} 20 \mathrm{f}^{\mathrm{ff}}$ or Vav-iCre ${ }^{-}$, Baf200 ${ }^{f /+}$ WT controls. Efficient deletion of Baf200 in the BM, spleen, and thymus cells from Vav-iCre
${ }^{+}$, Baf200 $0^{f f}$ mice was confirmed by genomic PCR (Additional file 1: Figure S6a). Peripheral blood counts revealed that $\mathrm{Vav}-\mathrm{iCr} \mathrm{C}^{+}$, Baf200 $0^{\mathrm{f} f f}$ mice exhibited decreased $\mathrm{RBC}$ number, hemoglobin concentration, hematocrit, and mean corpuscular hemoglobin along with increased RDW, suggesting a mild anemia may occur in $\mathrm{Vav}_{-i \mathrm{Cr}}{ }^{+}$, Baf200 $0^{f / f}$ mice (Fig. 4a and Additional file 1: Figure S6b). Consistently, $\mathrm{Vav}-\mathrm{iCr} \mathrm{C}^{+}$, Baf200 $\mathrm{O}^{f / f}$ mice exhibited slightly paler bone and splenomegaly (Fig. 4b). However, the total number of nucleated cells in the BM and spleen from Vav-iCre ${ }^{+}$, Baf $200^{f f}$ mice were comparable with those from WT mice (Fig. 4c). Flow cytometric analysis revealed impairment of erythropoiesis with decreased frequencies of EryB and EryC cells in the BM and increased frequencies of ProE and Ter119 ${ }^{\text {high }}$ cells in the spleen from 


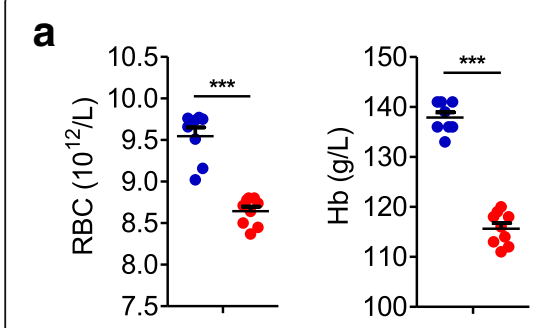

b
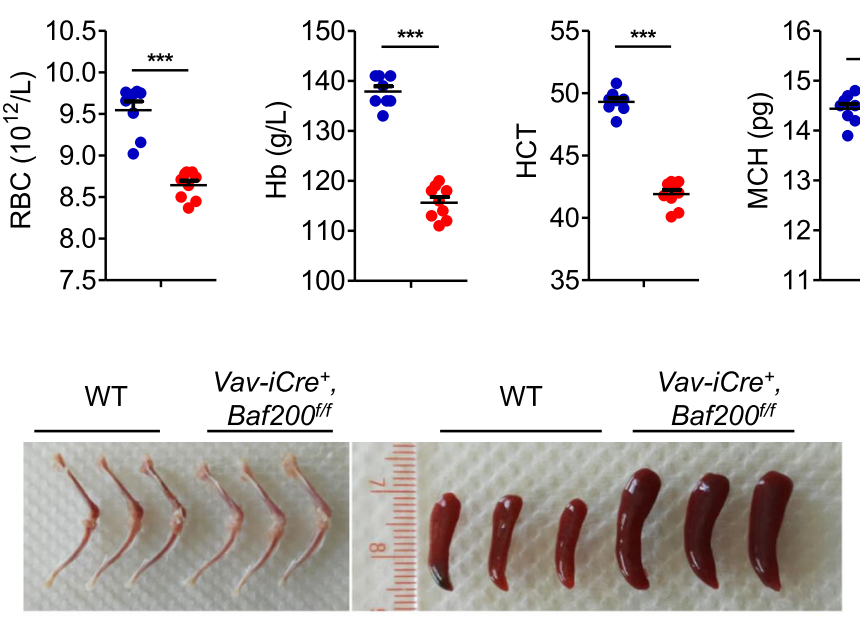
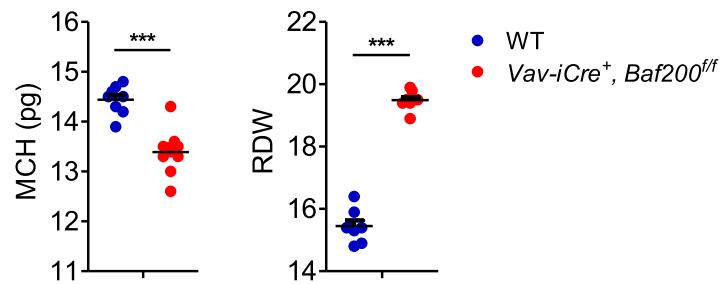

C

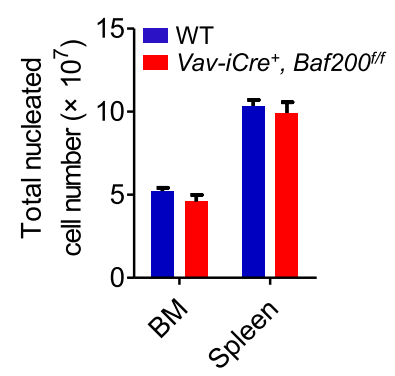

d

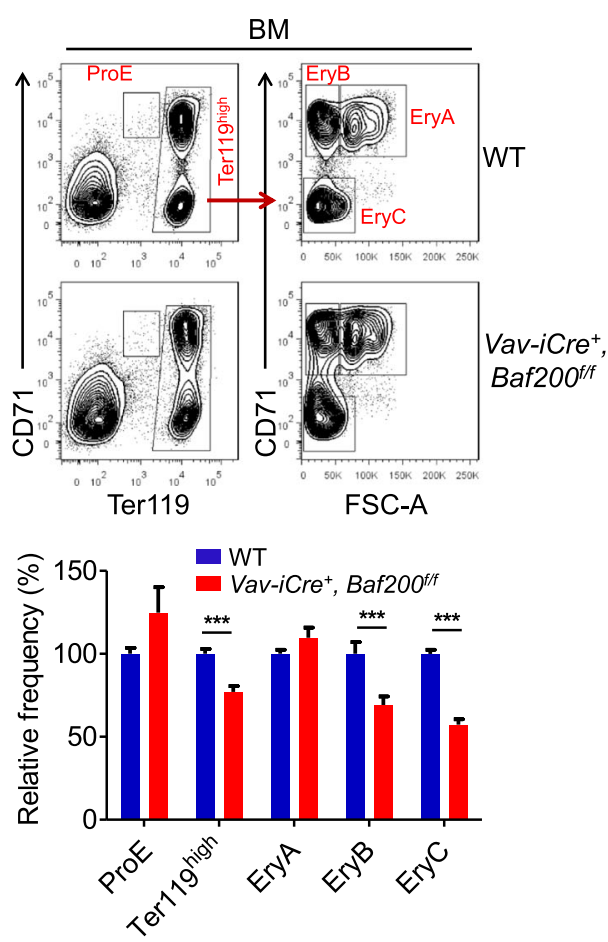

e
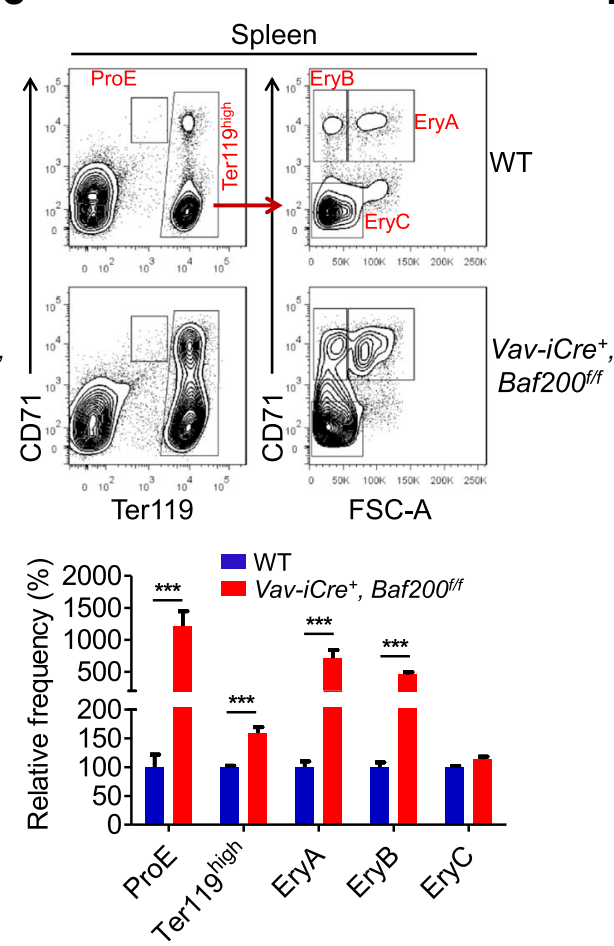

f
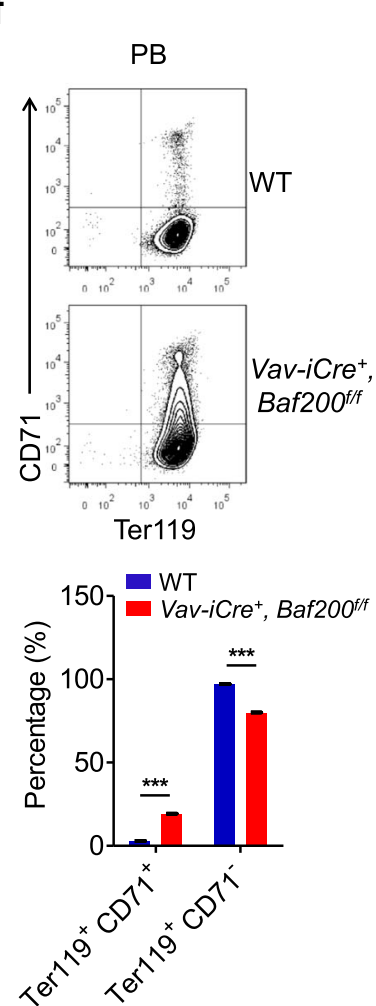

Fig. 4 Baf200 contributes to adult erythropoiesis. a Red blood cell parameters of peripheral blood from 10- to 12-week-old WT $(n=8)$ and Vav-iCre ${ }^{+}$, Baf200 $^{f f f}(n=9)$ mice. b Representative photographs of bone and spleen from 8-week-old WT and Vav-iCre ${ }^{+}$, Baf200 fff mice. c Graph showing the total nucleated cell number of BM and spleen from 8-week-old WT and Vav-iCre ${ }^{+}$, Baf200 fff mice ( $n=6$ per genotype). $\mathbf{d}$ Representative FACS profiles (top) and graph (bottom) showing relative frequency of CD71/Ter119 erythroid subsets in the BM from WT and Vav-iCre ${ }^{+}$, Baf200 ${ }^{f / f}$ mice $(n=6$ per genotype for each subset). e Representative FACS profiles (top) and graph (bottom) showing relative frequency of CD71/Ter119 erythroid subsets in the spleen from WT and Vav-iCre $e^{+}$Baf20f fff mice ( $n=6$ per genotype for each subset). f Representative FACS profiles (top) and graph (bottom) showing percentage of CD71/Ter119 erythroid subsets in the peripheral blood from WT and Vav-iCre ${ }^{+}$, Baf200 $0^{f / f}$ mice $(n=10$ per genotype for each subset). Data are shown as means \pm SEM. ${ }^{* * *} P<0.001$ 
Vav-iCre ${ }^{+}$, Baf200 $0^{f f}$ mice (Fig. $4 \mathrm{~d}$, e). The activation of erythropoiesis in the spleen might have been an attempt to compensate for insufficient erythropoiesis in the BM. Surprisingly, an increase of immature erythrocytes defined as Ter $119^{+} \mathrm{CD}_{71}{ }^{+}$and a decrease of mature erythrocytes defined as Ter $119^{+} \mathrm{CD} 71^{-}$were detected in the peripheral blood of $\mathrm{Vav}_{-i \mathrm{Cre}}^{+}$, Baf200 ${ }^{f / f}$ mice (Fig. 4f). Taken together, the results indicate that Baf200 contributes to adult erythropoiesis.

\section{Baf200 is involved in maintaining the adult hematopoiesis in steady state}

Flow cytometric analysis of the HSC compartments demonstrated decreases in the percentages and absolute numbers of LT-HSCs, ST-HSCs, MPP cells, common myeloid progenitor (CMP) cells, and MEP cells in the BM of $\mathrm{Vav}_{-i C r e^{+}}$, Baf200 $0^{f f f}$ mice (Fig. 5a, b). Enhanced extramedullary hematopoiesis in the spleen usually accompanies a decrease in $\mathrm{BM}$ hematopoiesis. Indeed, the percentages and numbers of CMP, GMP, and MEP cells in the spleen of $\mathrm{Vav}-\mathrm{iCre} \mathrm{C}^{+}, \mathrm{Baf} 200^{f / f}$ mice were increased (Fig. 5c, d), indicating that Baf200 deficiency promotes extramedullary hematopoiesis. Flow cytometric analysis found no significant differences in the cell cycle status or apoptosis of LSK cells in $\mathrm{Vav}-i \mathrm{Cre}^{+}, \mathrm{Baf2O0}^{\mathrm{f} / \mathrm{f}}$ mice and WT controls (Additional file 1: Figure S7a-g). The data reveal that Baf200 is not required for cell cycle process or apoptosis of the adult LSK compartment in steady-state hematopoiesis.

To determine the potential molecular pathways by which Baf200 regulates BM HSCs function, we conducted RNA-seq assay on purified BM LSK cells from $\mathrm{Vav}^{-i C r e}{ }^{+}, \mathrm{Baf}_{20} 00^{f / f}$ and WT mice. A total of 264 genes were upregulated and 198 genes were downregulated in Baf200-deficient LSK cells (Fig. 5e). Although the top GO terms are related to immune response, cell adhesion, and metabolic process, the erythrocyte development and hematopoiesis terms were also observed (Additional file 1: Figure S8). RT-qPCR experiment confirmed the altered expression of several hematopoiesis-associated genes (Fig. 5f), suggesting that Baf200 has an impact on adult HSC function, at least in part through regulation of the hematopoiesis-related genes.

\section{Cell-intrinsic Baf200 is required for HSC maintenance}

Competitive hematopoietic reconstitution assays were performed to further characterize the requirement of Baf200 for adult HSC maintenance by transplanting BM cells from $\mathrm{Vav}-i \mathrm{Cre}^{+}$, Baf200 $0^{f / f}$ or WT controls together with competitor BM cells into lethally irradiated recipients (Fig. 6a). Sixteen weeks post-transplantation, Baf200deficient HSCs failed to repopulate lymphoid and myeloid lineages in the peripheral blood, BM, spleen, and thymus of the recipients (Fig. 6b and
Additional file 1: Figure S9a). Moreover, the mutant donor-derived LSK cells were barely detectable in chimeras despite the comparable homing capacity of WT and Baf200-deficient BM cells (Fig. 6b and Additional file 1: Figure S9b). The results indicate that Baf200 is required for the maintenance of adult BM HSCs.

To further elucidate whether Baf200 regulates hematopoiesis in a cell-intrinsic manner, we firstly utilized inducible Baf200-deletion mice (Mx1-Cre $\left.B a f 200^{f / f}\right)$ and performed competitive transplantation experiments as above (Fig. 6c). Before poly(I:C) injection, Mx1-Cre ${ }^{+}$, Baf200 ${ }^{f f f}$ and WT BM cells had similar reconstitution capacity, while loss of Baf200 also led to gradual but significant decrease of lymphoid and myeloid lineages in the recipients (Fig. 6d). In addition, we transplanted CD45.1 $1^{+} \mathrm{BM}$ cells into lethally irradiated $\mathrm{Vav}-\mathrm{iCr} e^{+}, \mathrm{Baf} 200^{f / f}$ or WT mice. Two months later, BM cells from the recipients were harvested and cotransplanted into lethally irradiated recipients together with competitor cells. Sixteen weeks later, flow cytometry assays were performed to detect the lineage contribution of transplanted cells (Additional file 1: Figure S9c). The results revealed no difference in contribution ratios of any lineages (Additional file 1: Figure S9d). Therefore, these data demonstrate that Baf200 is a cell autonomously required for the adult HSC maintenance.

Considering that Tie2-Cre-mediated deletion of Baf200 led to embryonic lethality, we then detected the effects of Baf200 deletion on fetal hematopoiesis from Vav-iCre ${ }^{+}$, Baf $200^{f / f}$ mice. Although there was no difference in the absolute number of FL cells in E14.5 $\mathrm{Vav}_{-i C r e}{ }^{+}$, Baf200 f/f and WT embryos (Additional file 1: Figure S10a), detailed flow cytomeric analysis showed that there was a blockade of S3 to S4 transition during erythroid differentiation in the $\mathrm{Vav}-i \mathrm{Cre}^{+}, \mathrm{Baf} 20 \mathrm{f}^{f / f}$ embryos (Additional file 1: Figure S10b). The frequency and total number of LSK cells and LT-HSCs in the FL from $\mathrm{Vav}_{-i C r}{ }^{+}, \mathrm{Baf} 200^{f / f}$ embryos remained unchanged compared with those in the littermate controls (Additional file 1: Figure S10c). However, competitive transplantation assay demonstrated that FL cells from Vav-iCre ${ }^{+}$, Baf $200^{f f}$ embryos also showed impaired long-term reconstitution capacity (Additional file 1: Figure S10d-f).

\section{Loss of Baf200 accelerates leukemogenesis in MLL-AF9-induced AML}

The aforementioned results showed that Baf200 plays important roles in normal hematopoiesis; we then asked whether Baf200 also has a role in leukemogenesis. To test this, we utilized a previously described acute myeloid leukemia (AML) mouse model induced by MLL-AF9 fusion protein (Fig. 7a) [43]. Leukemia cells were harvested from two cohorts of primary recipients, and 
a
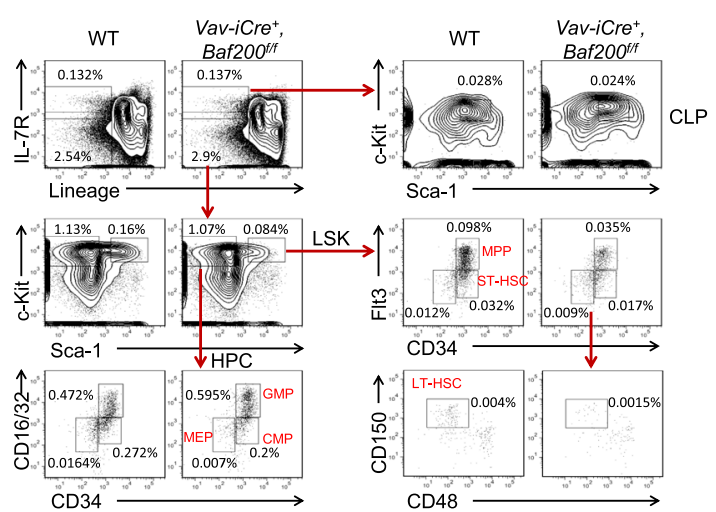

C

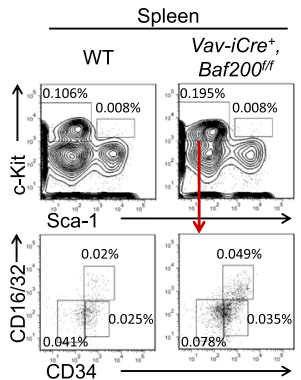

e

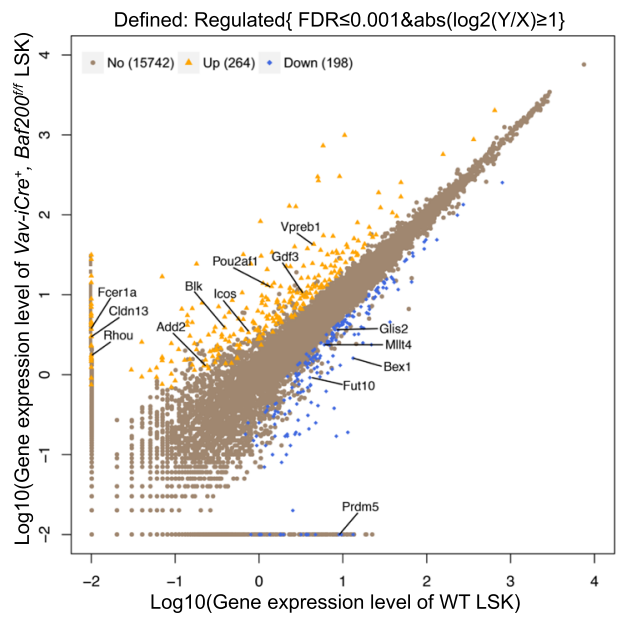

b

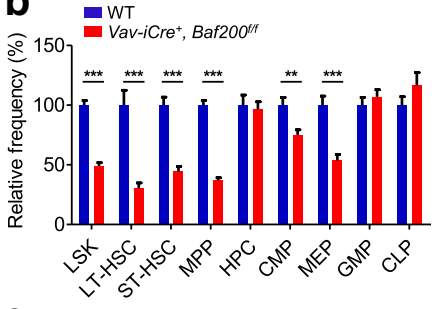

क्ष 100
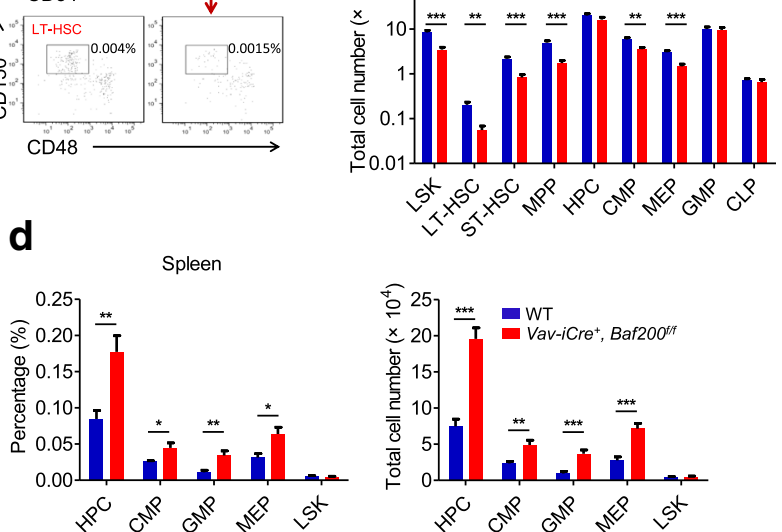

$\mathbf{f}$

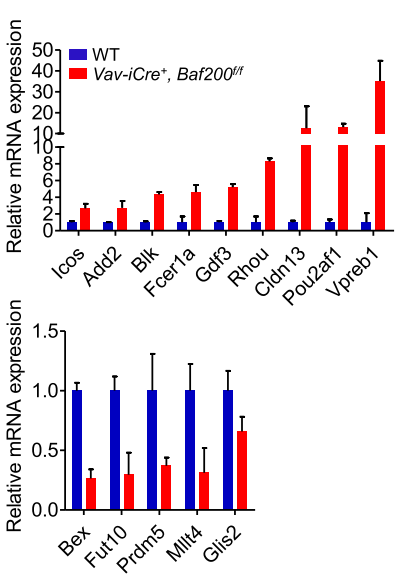

Fig. 5 Baf200 is required for adult hematopoiesis in steady state. a Representative FACS profiles showing hematopoietic stem and progenitor cells (HSPCS) in the BM from WT and Vav-iCre+, Baf200 f/f mice. b Graph showing the relative frequency (top) and absolute cell number (bottom) of the indicated subsets in the BM from WT and Vav-iCre ${ }^{+}$, Baf200 $0^{f / f}$ mice $(n=5-9$ per genotype for each subset). c Representative FACS profiles showing hematopoietic progenitors in the spleen from male WT and Vav-iCre+ ${ }^{+}$Baf200 fff mice (8-10-week-old). $\mathbf{d}$ Graph showing the percentage (left) and absolute cell number (right) of the indicated subsets in the spleen from male WT and Vav-iCre ${ }^{+}$, Baf200 ${ }^{f / f}$ mice $(8-10-$ week-old) $(n=6$ per genotype for each subset). e Scatter plots of all expressed genes in BM LSK cells from WT and Vav-iCre ${ }^{+}, B_{\text {Bf } 200^{f f f}}$ mice. Blue indicates downregulation gene, yellow indicates upregulation gene, and grey indicates non-regulation gene in Vav-iCre ${ }^{+}, B a f 200^{f / f}$ LSK cells. $\mathbf{f}$ Upregulation genes and downregulation genes in Vav-iCre+ ${ }^{+}$Baf200 ${ }^{f f f}$ LSK cells were confirmed by RT-qPCR ( $n=2$ per genotype). Data are shown as means \pm SEM. ${ }^{*} P<0.05 ;{ }^{* *} P<0.01 ;{ }^{* * *} P<0.001$

$50,000,200,000$, or 500,000 cells were transplanted into sublethally irradiated recipients in the secondary transplantation. Notably, Baf200 deficiency significantly accelerated the leukemia progression in the secondary transplantation. All mice in $\mathrm{Vav}-i \mathrm{Cre}^{+}, \mathrm{Baf} 200^{f / f}$ group died at around 25 days post-transplantation, whereas the average survival lifespan of recipients in WT group behaved in a dose-dependent manner (Fig. 7b). We then adopted 200,000 donor cells as a standard in the followup experiments. $\mathrm{Vav}-i \mathrm{Cre}^{+}$, Baf200 $0^{f / f}$ group mice showed 


\section{a}

Donor: CD45.2+ BM cells from WT or

Vav-iCre ${ }^{+}$, Baf200 $0^{f / f}$ mice

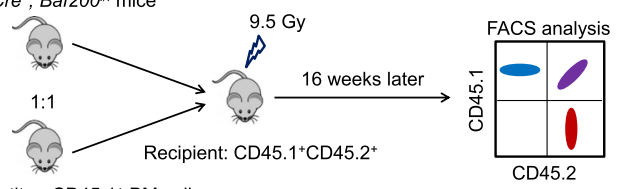

Competitor: CD45.1+ BM cells

C

Donor: CD45.2 ${ }^{+} \mathrm{BM}$ cells from WT or $\mathrm{Mx} 1-\mathrm{Cre}^{+}, \mathrm{Baf200^{f/f }}$ mice

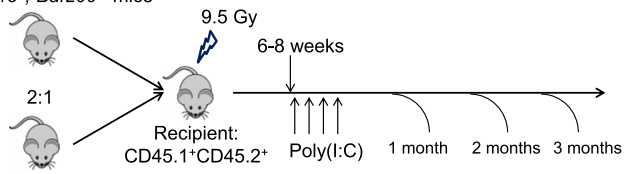

Competitor: CD45.1+ BM cells

d
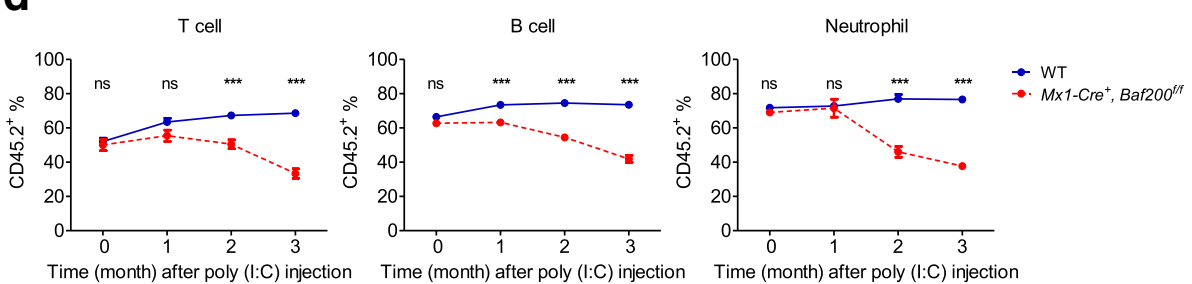

Fig. 6 Baf200-deficient BM HSCs have a significantly impaired long-term reconstitution capacity. a Scheme of competitive BM transplantation assay for WT and Vav-iCre ${ }^{+}$, Baf200 f/f mice. $\mathbf{b}$ The graph showing the relative ratios of CD45.2 versus CD45.1 of the indicated cell types in recipient mice 16 weeks after transplantation ( $n=7$ per genotype for each subset). c Scheme of competitive BM transplantation assay for WT and Mx 1 -Cre + , Baf200 f/f mice. d Percentage of donor-derived T cells (left), B cells (middle), and neutrophils (right) in the peripheral blood from recipient mice assessed at different time point ( $n=5$ per genotype for each subset). Data are shown as means \pm SEM. ${ }^{* * *} P<0.001$

much paler bones and splenomegaly at 20 days posttransplantation (Fig. 7c). Histology evaluation revealed more leukemia blasts in the peripheral blood, BM, spleen, and liver in $\mathrm{Vav}-\mathrm{iCre} \mathrm{C}^{+}$, Baf200 $\mathrm{f}^{\mathrm{ff}}$ group recipients than WT group (Fig. 7d), and flow cytometry confirmed much more $\mathrm{GFP}^{+}$leukemia cells in the peripheral blood, BM, and spleen from $\mathrm{Vav}-i \mathrm{Cre}^{+}, \mathrm{Baf200^{f/f }}$ group recipients (Fig. 7e). The $\mathrm{Vav}-\mathrm{iCre} \mathrm{C}^{+}$, Baf200 f/f group mice consistently exhibited increased myeloid cells and decreased lymphoid cells in the peripheral blood compared with WT mice (Fig. 7f, g). In addition, $\mathrm{Vav}-i \mathrm{Cre} e^{+}, \mathrm{Baf} 200^{f / f}$ group mice showed significantly accelerated accumulation of leukemia cells in the peripheral blood (Fig. 7h).

We then detected the leukemia stem cell (LSC) frequency in WT and $\mathrm{Vav}-\mathrm{iCre} \mathrm{C}^{+}$, Baf200 ${ }^{f / f}$ recipients and found that the percentage of LSC-enriched population $\left(\mathrm{GFP}^{+} \mathrm{Lin}^{-} \mathrm{C}-\mathrm{Kit}^{+}\right)$were much higher in the $\mathrm{BM}$ and spleen of $\mathrm{Vav}_{-i \mathrm{Cre}}^{+}$, Baf200 f/f group (Fig. 8a). Another set of markers for enriched LSCs confirmed an increase in the percentage of $\mathrm{GFP}^{+} \mathrm{Grl}^{-} \mathrm{c}-\mathrm{Kit}^{+}$cells in $\mathrm{Vav}-i \mathrm{Cr} e^{+}$, Baf200 fff group (Fig. 8b). To explore the underlying mechanisms by which Baf200 deficiency accelerates the leukemia progression, we sorted $\mathrm{GFP}^{+} \mathrm{BM}$ leukemia cells from $\mathrm{Vav}-i \mathrm{Cre}^{+}$, Baf200 $0^{f / f}$ and WT group and detected the expression of hematopoiesis-related genes by
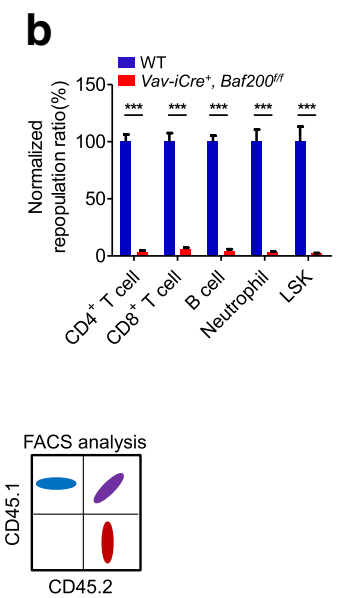

RT-qPCR assay. Interestingly, several genes associated with hematopoietic malignancies, including Gata2, Cebpa, Pdk1, Myb, Myc, and Meis1 [44-48], were upregulated in $\mathrm{Vav}^{-i \mathrm{Cre}^{+}}$, Baf200 $0^{f / f}$ AML cells (Fig. 8c). The expression of Cebpe, a key transcription factor in granulopoiesis [49], was decreased in $\mathrm{Vav}-i \mathrm{Cre}^{+}$, Baf200 $0^{\mathrm{f} f}$ AML cells (Fig. 8d). Furthermore, loss of Baf200 promotes the progression of leukemogenesis partially by inhibiting the expression of $p 57$ and $p 16$ (Fig. 8d). Taken together, the results indicate that Baf200 acts as a tumor suppressor in MLL-AF9-induced AML.

\section{Discussion}

Although ATP-dependent SWI/SNF complex have been demonstrated to play pivotal roles in normal or malignant hematopoiesis [13-21], little is known about the function of PBAF-specific subunit Baf200 in these processes. Here, we utilized several hematopoietic lineagespecific Cre mouse lines to investigate the role of Baf200 in fetal and adult hematopoiesis, as well as in leukemogenesis. Our current study clearly showed the cell-intrinsic requirement of Baf200 in hematopoietic cells, as FL HSCs from Tie2-Cre ${ }^{+}, \mathrm{Baf}_{200} \mathrm{O}^{f f f}$ or $\mathrm{Vav}-i \mathrm{Cr} e^{+}$, Baf200 f/f embryos and BM HSCs from $\mathrm{Vav}_{-i C r}{ }^{+}$, 
a
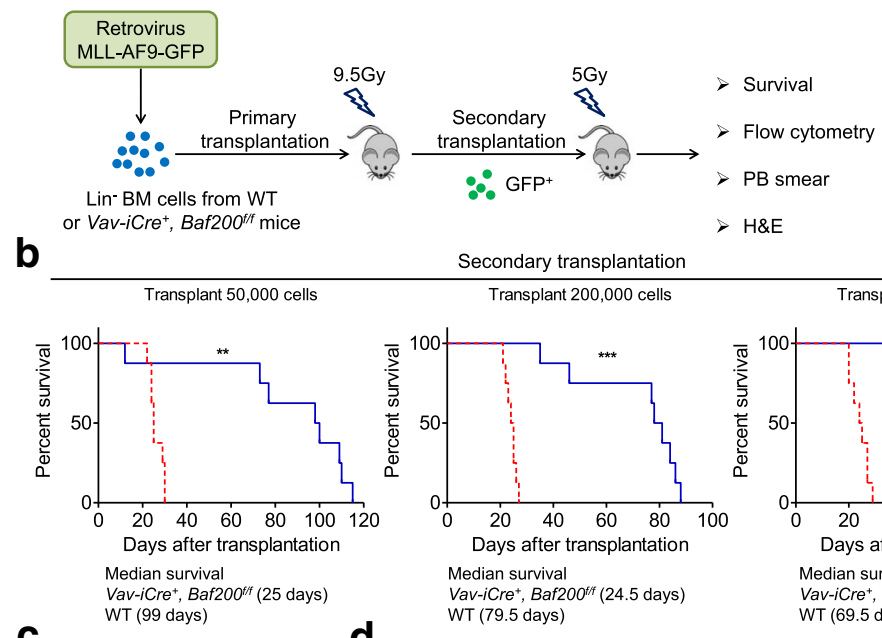

。

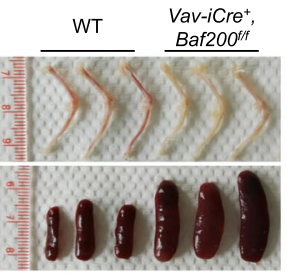

C. Secondary transplantation

$H \& E$

$\begin{array}{cc}\text { Secondary transplantation } & \\ \text { Transplant } 200,000 \text { cells } & \text { Transplant } 500,000 \text { cells }\end{array}$
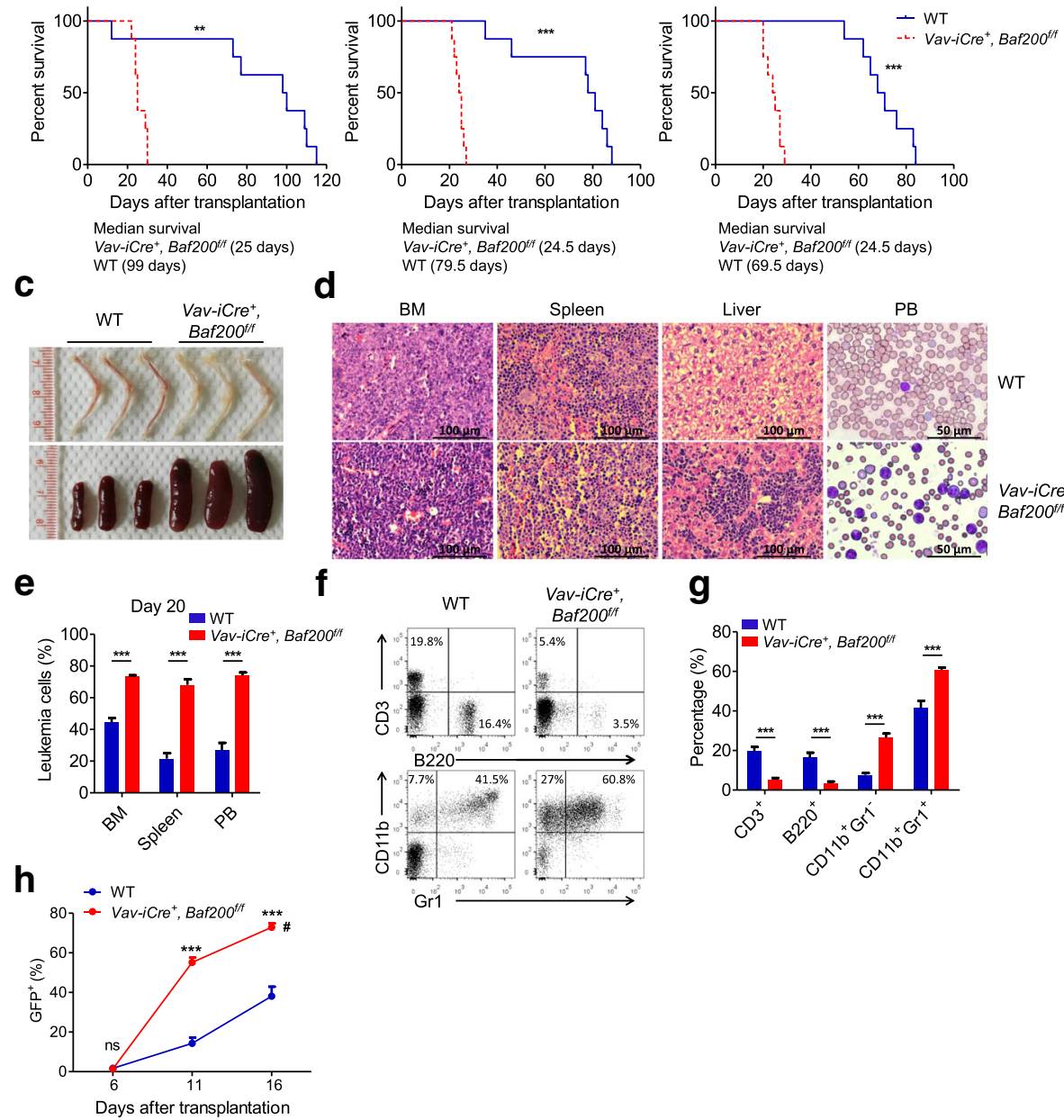

WT (69.5 days)
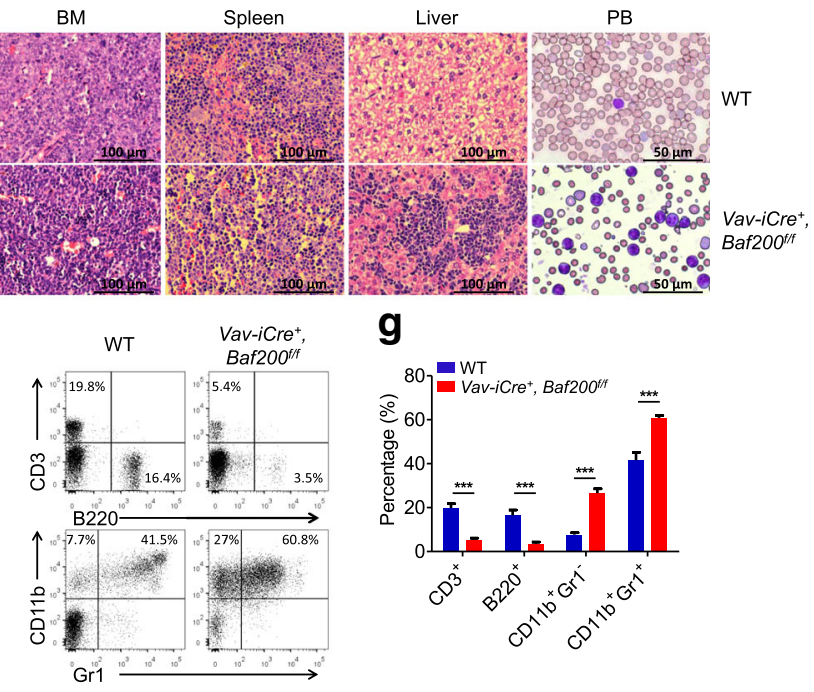

g

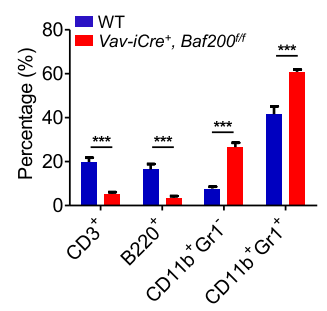

Fig. 7 Loss of Baf200 accelerates leukemogenesis in MLL-AF9-induced AML. a Scheme to investigate the role of endogenous Baf200 in MLL-AF9-induced AML. b Survival curve of recipient mice engrafted with leukemia cells from primary recipients in the secondary transplantation assay ( $n=8$ per genotype). c Representative photos of bone and spleen from recipient mice. $\mathbf{d}$ Histology of the BM, spleen, and liver and blood smear from recipients. e Graph showing the percentage of leukemia cells in the BM ( $n=3$ per genotype), spleen ( $n=3$ per genotype), and PB (peripheral blood) ( $n=8$ per genotype) from recipient mice. $\mathbf{f}$ Representative flow profiles of myeloid and lymphoid subsets in the PB from recipients. $\mathbf{g}$ Graph showing the percentage of the indicated subsets in the PB ( $n=8$ per genotype for each subset). $\mathbf{h}$ Graph showing the percentage of leukemia cells $\left(\mathrm{GFP}^{+}\right)$in the PB from recipients at indicated days post-transplantation ( $n=5$ per genotype for each subset). Vav-iCre ${ }^{+}$, Baf200/f group mice showed accelerated accumulation of leukemia cells in the PB. The number sign indicates Vav-iCre ${ }^{+}$, Baf200/f/f group mice started to die at 16 days posttransplantation. Data are shown as means \pm SEM. ${ }^{* *} P<0.01 ;{ }^{* * *} P<0.001$

Baf200 $0^{f / f}$ mice exhibited impaired capacity of long-term hematopoietic reconstitution. Moreover, we utilized adoptive transfer strategy with inducible Mx1-Cre system and verified the cell-intrinsic requirement for Baf200 in the FL or adult BM HSC maintenance.
We noticed that Tie2-Cre ${ }^{+}$, Baf200 fff mice displayed much more severe phenotypes than $\mathrm{Vav}-i \mathrm{Cr} \mathrm{e}^{+}, \mathrm{Baf} 200^{\mathrm{f} / \mathrm{f}}$ mice. Tie2-Cre $e^{+}$, Baf200 fff embryos died at embryonic stage with defective fetal erythropoiesis, whereas $\mathrm{Vav}_{-i C r e}^{+}$, Baf200ff mice were born at expected 
a

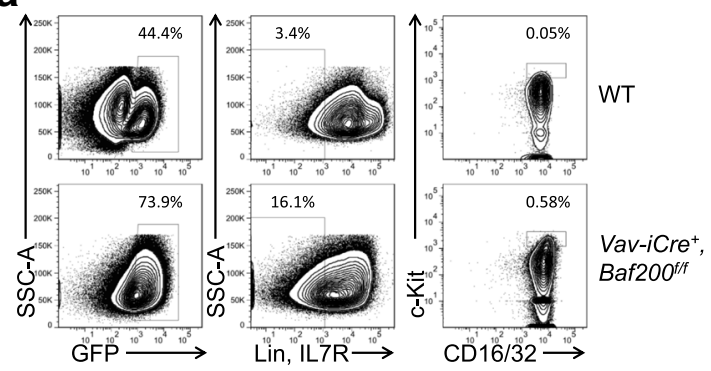

b

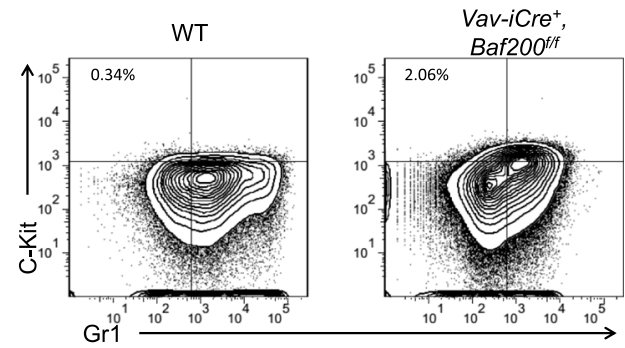

C

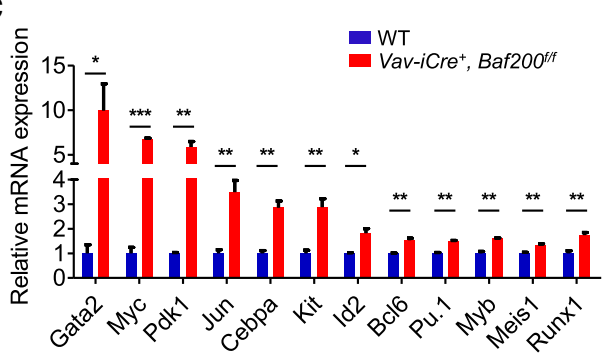

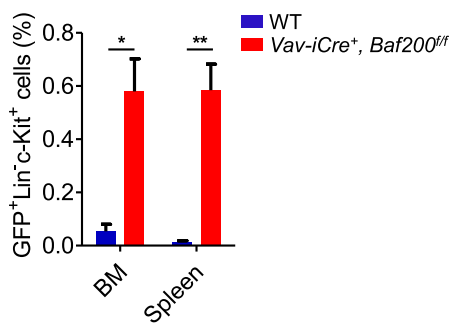

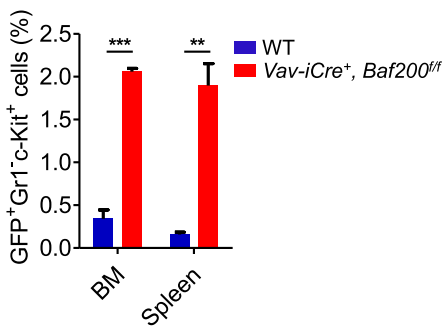

d

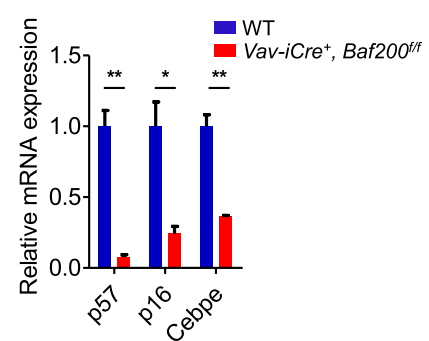

Fig. 8 Increased percentage of LSCs in Baf200-deficient AML. a The flow profiles (left) and graph (right) showing the percentage of LSCS (GFP' Lin ${ }^{-} \mathrm{c}-K i t$ $\left.{ }^{+}\right)$in the BM from recipients ( $n=3$ per genotype). $\mathbf{b}$ The flow profiles (left) and graph (right) showing the percentage of GFP ${ }^{+} \mathrm{Gr}^{-}{ }^{-} \mathrm{c}-\mathrm{Kit}^{+}$subset in the BM from recipients ( $n=3$ per genotype). c, d Upregulation (c) and downregulation (d) genes in Vav-iCre ${ }^{+}$, Baf200 ${ }^{f / f}$ leukemia cells $(n=3$ per genotype). Data are shown as means \pm SEM. ${ }^{*} P<0.05 ;{ }^{* *} P<0.01 ;{ }^{* *} P<0.001$

Mendelian ratio with an appearance indistinguishable from their littermate controls. Tie2-Cre is originally expressed in hemogenic endothelium as early as E6.5, which leads to efficient deletion in endothelial cells and their progeny (HSCs and all definitive blood cells) $[31,50]$. In contrast, $\mathrm{Vav}-\mathrm{iCre}$ begins to be expressed at E9.5 and the expression was greatly enhanced at E12.5; thus, it causes high deletion efficiency in hematopoietic lineages but low deletion efficiency in endothelial cells $[42,51]$. So we speculated one possibility is that Baf200 in endothelial cells may also play a role in fetal hematopoiesis. Endothelial cell-specific Cre strain, such as VE-Cadherin-CreERT2, would be useful for dissecting the precise role of Baf200 in endothelial cells in the future. In mouse, there are two waves of fetal hematopoiesis, primitive, and definitive. Primitive hematopoiesis produces erythroid and myeloid cells in the YS blood island at around E7.5. Definitive hematopoietic cells (transient erythroid and myeloid progenitors) arise from hemogenic endothelium in the YS at around E8.25, and these cells can migrate into the FL to give rise to erythroid and myeloid progeny. Generation of de novo HSCs takes place in the AGM region at around E10.5, soon the HSCs migrate into the FL, expand, and differentiate [1, 52]. Previous study revealed that Tie2-Cre mediates recombination in almost all primitive and definitive hematopoietic cells [50], while only definitive hematopoietic cells that have already homed into FL can express recombinase in Vav-iCre strain [42]. Therefore, another possibility of the distinct phenotypes observed in Tie2-Cre and Vav-iCre strain is the different timing of Cre activity.

Several SWI/SNF complex subunits have been reported to play important roles in hematopoiesis. Brg1, a catalytic subunit of the BAF and PBAF complexes, is involved in both primitive and definitive erythropoiesis $[13,14]$. Baf250a, a subunit of the BAF complex, controls the pool size of FL HSCs [20]. Baf53a, a subunit of both BAF and the PBAF complex, is required for HSC 
maintenance [18]. Baf180, another unique subunit of PBAF complex, is required for long-term reconstitution potential of HSCs [22]. Our current study provided additional evidence that Baf200, a PBAF specific subunit, plays a key role in hematopoiesis. Although Baf200 and Baf180 are both the PBAF-specific subunits, Baf200 seems to have a more important role than Baf180 in hematopoiesis. Baf200-deficient HSCs were poorly competitive in long-term hematopoietic reconstitution following primary transplantation, whereas Baf180-deficient HSCs had a compromised long-term reconstitution potential only in secondary transplantation. BAF200 interacts with BAF180 and some other identical subunits within PBAF complex to exert their function [12]. However, a previous study showed that BAF200, but not BAF180, is essential for the stability of PBAF complex [53]. This may be an explanation for the appeared more vital role of Baf200 in hematopoiesis. However, whether Baf200 regulates hematopoiesis through a PBAF-independent mechanism needs to be determined in future studies.

Epigenetic modifiers may have adverse effects on normal and malignant hematopoiesis. For example, Asxl1, a polycomb group protein, is essential for preserving hematopoietic reconstitution capacity, whereas haploinsufficiency of Asxl1 can cause MDS-like disease [54, 55]. Utx, a di- and trimethyl H3K27 demethylase, is required for normal hematopoiesis [56]; meanwhile, Utx serves as a tumor suppressor in NOTCH1-induced $\mathrm{T}$ cell acute lymphoblastic leukemia (T-ALL) [57]. Moreover, differentially expressed genes regulated by Utx were found in normal Utx-deficient BM cells or in Utx-deficient leukemia cells $[56,57]$, indicating Utx may regulate the target genes in a dual context-dependent manner. Our current study found that Baf200 deficiency impairs normal hematopoiesis but accelerates MLL-AF9-driven leukemogenesis, suggesting that Baf200 may play contrast roles in normal and malignant hematopoiesis. We also revealed diverse genes regulated by Baf200 in normal LSK cells and AML cells. However, whether Baf200 regulate its target genes or pathways in a dual context-dependent manner in normal or leukemia cells still need further investigation.

Several potential Baf200 targets were identified by RNA-seq analysis and RT-qPCR in our study. RNA-seq results revealed diverse patterns of gene differentially regulated by Baf200 in different cell lineages, including FL LSK, FL erythrocytes, adult BM LSK, and leukemia cells. These results indicate that Baf200 may regulate different subsets of target genes in a cell contextdependent manner. It may recruit different transcription factors or histone modifiers or be recruited by them to the specific target genes in different cell types. Whether Baf200 controls these genes through a direct or indirect manner still needs further investigations. Future studies will aim to explore the underlying mechanisms of Baf200 in normal and malignant hematopoiesis by using ChIP-based analysis.

\section{Conclusions}

In summary, this study reveals that loss of Baf200 impairs the normal fetal and adult hematopoiesis while accelerates the progression of MLL-AF9-induced leukemia, which provide a potential therapeutic target for leukemia treatment.

\section{Additional file}

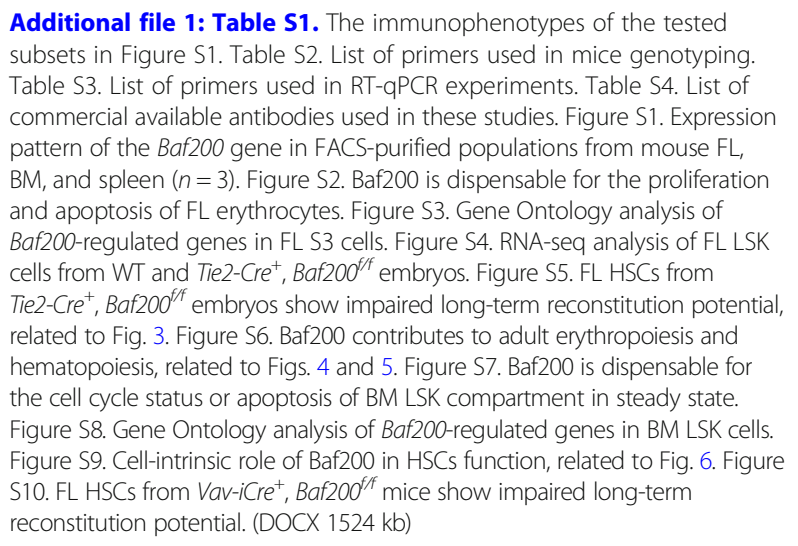

\section{Abbreviations}

AGM: Aorta-gonad-mesonephros; AML: Acute myeloid leukemia; ATP: Adenosine triphosphate; BAF: BRG1-associated factor; BM: Bone marrow; CMP: Common myeloid progenitor; FL: Fetal liver; GMP: Granulocyte-macrophage progenitor; GO: Gene Ontology; HSC: Hematopoietic stem cell;

HSPCS: Hematopoietic stem and progenitor cells; LSC: Leukemic stem cell; LSK: Lineage ${ }^{-} \mathrm{SCal}^{+}{ }^{+} \mathrm{C}-\mathrm{Kit}^{+}{ }^{+}$; LT-HSC: Long-term hematopoietic stem cell; MDS: Myelodysplastic syndrome; MEP: Megakaryocyte-erythroid progenitor; MPP: Multipotent progenitor; PBAF: Polybromo BRG1-associated factor; ProE: Pro-erythroblast; RT-qPCR: Reverse transcription-quantitative polymerase chain reaction; ST-HSC: Short-term hematopoietic stem cell; WT: Wild-type; YS: Yolk sac

\section{Acknowledgements}

We thank Dr. So Chi Wai Eric for providing the MSCV-MLL-AF9 plasmid. We thank Dr. Jianmin Wang, Dr. Jinyong Wang, Dr. Shengzhong Duan, and Dr. Lijian Hui for providing the CD45. $1^{+}$mice, Vav-iCre mice, Tie2-Cre mice, and Mx1-Cre mice, respectively. We thank Dr. Tao Cheng for the help in the leukemia mouse model. We acknowledge Dr. Jiang Zhu, Dr. Weijun Pan, Dr. Bo Zhou, Dr. Xiaoming Zhang, and Dr. Haibing Zhang for the valuable discussions.

\section{Funding}

This study was supported by the National Basic Research Program of China (2015CB964900) and the National Natural Science Foundation of China (31670906, 31471207, 81270618, and 81370601).

Availability of data and materials

All data generated in this study are included in the present article [and its supplementary information files].

\section{Author's contributions}

$L L$ and $Y Z$ designed the experiments, analyzed the data, and wrote the manuscript. LL and XW performed all the experiments. $X Z$ analyzed the RNA-seq data. PZ, WZ, HH, XY, and XD contributed to the mouse 
experiments. RZ contributed to the flow cytometry experiments. GM, HX, FM, $\mathrm{HH}$, and XS assisted with the manuscript preparation. BZ, SX, and YZ conducted the research. All authors read and approved the final manuscript.

\section{Ethics approval}

Animal protocols are reviewed and approved by the Institutional Animal Care and Use Committee (IACUC) in the Institut Pasteur of Shanghai.

\section{Consent for publication}

Not applicable

\section{Competing interests}

The authors declare that they have no competing interests.

\section{Publisher's Note}

Springer Nature remains neutral with regard to jurisdictional claims in published maps and institutional affiliations.

\begin{abstract}
Author details
${ }^{1}$ Institute of Biology and Medical Sciences, Soochow University, No. 199 Ren'ai Rd, Suzhou, China. ${ }^{2}$ Key Laboratory of Molecular Virology and Immunology, Institut Pasteur of Shanghai, Chinese Academy of Sciences, 320 Yue-Yang Road, Shanghai, China. ${ }^{3}$ University of Chinese Academy of Sciences, Beijing, China. ${ }^{4}$ The State Key Laboratory of Cell Biology, CAS Center for Excellence in Molecular Cell Science, Shanghai Institute of Biochemistry and Cell Biology, Chinese Academy of Sciences, 320 Yue-Yang Road, Shanghai, China. ${ }^{5}$ CAS-MPG Partner Institute for Computational Biology, Shanghai Institutes for Biological Sciences, Chinese Academy of Sciences, Shanghai, China. ${ }^{6}$ Institute of Blood Transfusion, Chinese Academy of Medical Sciences and Peking Union Medical College, Chengdu, China. ${ }^{7}$ School of Life Sciences, Shanghai University, Shanghai, China. ${ }^{8}$ Bone Marrow Transplantation Center, the First Affiliated Hospital, Zhejiang University School of Medicine, Hangzhou, China. 'Department of Hematology, Shanghai Jiao Tong University Affiliated Shanghai General Hospital, Shanghai, China.
\end{abstract}

Received: 24 November 2017 Accepted: 5 February 2018 Published online: 26 February 2018

\section{References}

1. Orkin SH, Zon LI. Hematopoiesis: an evolving paradigm for stem cell biology. Cell. 2008;132(4):631-44.

2. Porcher $\mathrm{C}$, Chagraoui $\mathrm{H}$, Kristiansen MS. SCL/TAL1: a multifaceted regulator from blood development to disease. Blood. 2017;129(15):2051-60

3. de Bruijn M, Dzierzak E. Runx transcription factors in the development and function of the definitive hematopoietic system. Blood. 2017;129(15):2061-9.

4. Sood R, Kamikubo Y, Liu P. Role of RUNX1 in hematological malignancies. Blood. 2017;129(15):2070-82.

5. Avellino R, Delwel R. Expression and regulation of C/EBPalpha in normal myelopoiesis and in malignant transformation. Blood. 2017;129(15):2083-91.

6. Katsumura KR, Bresnick EH, Group GFM. The GATA factor revolution in hematology. Blood. 2017;129(15):2092-102.

7. Crispino JD, Horwitz MS. GATA factor mutations in hematologic disease. Blood. 2017:129(15):2103-10

8. Yang W, Ernst P. Distinct functions of histone $\mathrm{H3}$, lysine 4 methyltransferases in normal and malignant hematopoiesis. Curr Opin Hematol. 2017;24(4): 322-8.

9. Kunimoto $\mathrm{H}$, Nakajima $\mathrm{H}$. Epigenetic dysregulation of hematopoietic stem cells and preleukemic state. Int J Hematol. 2017;106(1):34-44.

10. Rasko JE, Wong JJ. Nuclear microRNAs in normal hemopoiesis and cancer. J Hematol Oncol. 2017;10(1):8.

11. Tian X, Tian J, Tang X, Ma J, Wang S. Long non-coding RNAs in the regulation of myeloid cells. J Hematol Oncol. 2016;9(1):99.

12. Masliah-Planchon J, Bieche I, Guinebretiere JM, Bourdeaut F, Delattre O. SWI/SNF chromatin remodeling and human malignancies. Annu Rev Pathol. 2015;10:145-71.

13. Bultman SJ, Gebuhr TC, Magnuson T. A Brg1 mutation that uncouples ATPase activity from chromatin remodeling reveals an essential role for SWI/SNF-related complexes in beta-globin expression and erythroid development. Genes Dev. 2005;19(23):2849-61.
14. Griffin CT, Brennan J, Magnuson T. The chromatin-remodeling enzyme BRG1 plays an essential role in primitive erythropoiesis and vascular development. Development. 2008;135(3):493-500.

15. Gebuhr TC, Kovalev GI, Bultman S, Godfrey V, Su L, Magnuson T. The role of Brg1, a catalytic subunit of mammalian chromatin-remodeling complexes, in T cell development. J Exp Med. 2003;198(12):1937-49.

16. Shi J, Whyte WA, Zepeda-Mendoza CJ, Milazzo JP, Shen C, Roe JS, et al. Role of SWI/SNF in acute leukemia maintenance and enhancer-mediated Myc regulation. Genes Dev. 2013;27(24):2648-62.

17. Buscarlet M, Krasteva V, Ho L, Simon C, Hebert J, Wilhelm B, et al. Essential role of BRG, the ATPase subunit of BAF chromatin remodeling complexes, in leukemia maintenance. Blood. 2014;123(11):1720-8.

18. Krasteva V, Buscarlet M, Diaz-Tellez A, Bernard MA, Crabtree GR, Lessard JA The BAF53a subunit of SWI/SNF-like BAF complexes is essential for hemopoietic stem cell function. Blood. 2012;120(24):4720-32.

19. Krasteva V, Crabtree GR, Lessard JA. The BAF45a/PHF10 subunit of SWI/SNFlike chromatin remodeling complexes is essential for hematopoietic stem cell maintenance. Exp Hematol. 2017;48:58-71.e15.

20. Krosl J, Mamo A, Chagraoui J, Wilhelm BT, Girard S, Louis I, et al. A mutant allele of the Swi/Snf member BAF250a determines the pool size of fetal liver hemopoietic stem cell populations. Blood. 2010;116(10):1678-84.

21. Vradii D, Wagner S, Doan DN, Nickerson JA, Montecino M, Lian JB, et al. Brg1, the ATPase subunit of the SWI/SNF chromatin remodeling complex, is required for myeloid differentiation to granulocytes. J Cell Physiol. 2006; 206(1):112-8.

22. Lee $H$, Dai $F$, Zhuang $L$, Xiao ZD, Kim J, Zhang Y, et al. BAF180 regulates cellular senescence and hematopoietic stem cell homeostasis through p21. Oncotarget. 2016;7(15):19134-46.

23. Li M, Zhao H, Zhang X, Wood LD, Anders RA, Choti MA, et al. Inactivating mutations of the chromatin remodeling gene ARID2 in hepatocellular carcinoma. Nat Genet. 2011:43(9):828-9.

24. Zhao $H$, Wang J, Han $Y$, Huang Z, Ying J, Bi X, et al. ARID2: a new tumor suppressor gene in hepatocellular carcinoma. Oncotarget. 2011; 2(11):886-91.

25. Manceau G, Letouze E, Guichard C, Didelot A, Cazes A, Corte H, et al. Recurrent inactivating mutations of ARID2 in non-small cell lung carcinoma. Int J Cancer. 2013;132(9):2217-21.

26. He L, Tian $X$, Zhang $H$, Hu T, Huang $X$, Zhang $L$, et al. BAF200 is required for heart morphogenesis and coronary artery development. PLoS One. 2014; 9(10):e109493.

27. Sakai H, Hosono N, Nakazawa H, Przychodzen B, Polprasert C, Carraway HE, et al. A novel genetic and morphologic phenotype of ARID2-mediated myelodysplasia. Leukemia. 2017. https://doi.org/10.1038/leu.2017.319.

28. Cheng H, Hao S, Liu Y, Pang Y, Ma S, Dong F, et al. Leukemic marrow infiltration reveals a novel role for Egr3 as a potent inhibitor of normal hematopoietic stem cell proliferation. Blood. 2015;126(11):1302-13.

29. Huang da W, Sherman BT, Lempicki RA. Bioinformatics enrichment tools: paths toward the comprehensive functional analysis of large gene lists. Nucleic Acids Res. 2009;37(1):1-13.

30. Huang da W, Sherman BT, Lempicki RA. Systematic and integrative analysis of large gene lists using DAVID bioinformatics resources. Nat Protoc. 2009: 4(1):44-57.

31. Constien R, Forde A, Liliensiek B, Grone HJ, Nawroth P, Hammerling G, et al. Characterization of a novel EGFP reporter mouse to monitor Cre recombination as demonstrated by a Tie2 Cre mouse line. Genesis. 2001; 30(1):36-44.

32. Wong P, Hattangadi SM, Cheng AW, Frampton GM, Young RA, Lodish HF. Gene induction and repression during terminal erythropoiesis are mediated by distinct epigenetic changes. Blood. 2011;118(16):e128-38.

33. Lyon JJ, Watson RJ. Conditional inhibition of erythroid differentiation by CMyb/oestrogen receptor fusion proteins. Differentiation. 1995:59(3):171-8.

34. Cuddihy AE, Brents LA, Aziz N, Bender TP, Kuehl WM. Only the DNA binding and transactivation domains of c-Myb are required to block terminal differentiation of murine erythroleukemia cells. Mol Cell Biol. 1993;13(6): 3505-13.

35. Garcia-Santos D, Schranzhofer M, Horvathova M, Jaberi MM, Bogo Chies JA, Sheftel $A D$, et al. Heme oxygenase 1 is expressed in murine erythroid cells where it controls the level of regulatory heme. Blood. 2014;123(14):2269-77.

36. Esteghamat F, van Dijk TB, Braun H, Dekker S, van der Linden R, Hou J, et al. The DNA binding factor Hmg20b is a repressor of erythroid differentiation. Haematologica. 2011;96(9):1252-60. 
37. Lowell CA, Niwa M, Soriano P, Varmus HE. Deficiency of the Hck and Src tyrosine kinases results in extreme levels of extramedullary hematopoiesis. Blood. 1996;87(5):1780-92.

38. Dai XM, Ryan GR, Hapel AJ, Dominguez MG, Russell RG, Kapp S, et al. Targeted disruption of the mouse colony-stimulating factor 1 receptor gene results in osteopetrosis, mononuclear phagocyte deficiency, increased primitive progenitor cell frequencies, and reproductive defects. Blood. 2002; 99(1):111-20.

39. Pepler L, Yu P, Dwivedi DJ, Trigatti BL, Liaw PC. Characterization of mice harboring a variant of EPCR with impaired ability to bind protein C: novel role of EPCR in hematopoiesis. Blood. 2015;126(5):673-82.

40. Holmfeldt P, Ganuza M, Marathe H, He B, Hall T, Kang G, et al. Functional screen identifies regulators of murine hematopoietic stem cell repopulation. J Exp Med. 2016;213(3):433-49.

41. Campbell MR, Karaca M, Adamski KN, Chorley BN, Wang X, Bell DA. Novel hematopoietic target genes in the NRF2-mediated transcriptional pathway. Oxidative Med Cell Longev. 2013;2013:120305.

42. Chen MJ, Yokomizo T, Zeigler BM, Dzierzak E, Speck NA. Runx 1 is required for the endothelial to haematopoietic cell transition but not thereafter. Nature. 2009;457(7231):887-91.

43. Krivtsov AV, Twomey D, Feng Z, Stubbs MC, Wang Y, Faber J, et al. Transformation from committed progenitor to leukaemia stem cell initiated by MLL-AF9. Nature. 2006;442(7104):818-22.

44. Ohlsson E, Schuster MB, Hasemann M, Porse BT. The multifaceted functions of C/EBPalpha in normal and malignant haematopoiesis. Leukemia. 2016; 30(4):767-75.

45. Vicente C, Conchillo A, Garcia-Sanchez MA, Odero MD. The role of the GATA2 transcription factor in normal and malignant hematopoiesis. Crit Rev Oncol Hematol. 2012;82(1):1-17.

46. Lawrence HJ, Rozenfeld S, Cruz C, Matsukuma K, Kwong A, Komuves L, et al. Frequent co-expression of the HOXA9 and MEIS1 homeobox genes in human myeloid leukemias. Leukemia. 1999;13(12):1993.

47. Gonda TJ, Metcalf D. Expression of myb, myc and fos proto-oncogenes during the differentiation of a murine myeloid leukaemia. Nature. 1984; 310(5974):249-51.

48. Hu T, Li C, Zhang Y, Wang L, Peng L, Cheng H, et al. Phosphoinositidedependent kinase 1 regulates leukemia stem cell maintenance in MLL-AF9induced murine acute myeloid leukemia. Biochem Biophys Res Commun. 2015;459(4):692-8.

49. Yamanaka R, Barlow C, Lekstrom-Himes J, Castilla LH, Liu PP, Eckhaus M, et al. Impaired granulopoiesis, myelodysplasia, and early lethality in CCAAT/ enhancer binding protein epsilon-deficient mice. Proc Natl Acad Sci U S A. 1997;94(24):13187-92.

50. Tang $Y$, Harrington A, Yang $X$, Friesel RE, Liaw L. The contribution of the Tie2+ lineage to primitive and definitive hematopoietic cells. Genesis. 2010; 48(9):563-7.

51. Lan Y, He W, Li Z, Wang Y, Wang J, Gao J, et al. Endothelial Smad4 restrains the transition to hematopoietic progenitors via suppression of ERK activation. Blood. 2014;123(14):2161-71.

52. Ciau-Uitz A, Monteiro R, Kirmizitas A, Patient R. Developmental hematopoiesis: ontogeny, genetic programming and conservation. Exp Hematol. 2014;42(8):669-83.

53. Yan Z, Cui K, Murray DM, Ling C, Xue Y, Gerstein A, et al. PBAF chromatinremodeling complex requires a novel specificity subunit, BAF200, to regulate expression of selective interferon-responsive genes. Genes Dev. 2005;19(14):1662-7.

54. Abdel-Wahab O, Gao J, Adli M, Dey A, Trimarchi T, Chung YR, et al. Deletion of Asxl1 results in myelodysplasia and severe developmental defects in vivo. J Exp Med. 2013;210(12):2641-59.

55. Wang J, Li Z, He Y, Pan F, Chen S, Rhodes S, et al. Loss of Asxl1 leads to myelodysplastic syndrome-like disease in mice. Blood. 2014;123(4):541-53.

56. Thieme S, Gyarfas T, Richter C, Ozhan G, Fu J, Alexopoulou D, et al. The histone demethylase UTX regulates stem cell migration and hematopoiesis. Blood. 2013;121(13):2462-73.

57. Ntziachristos P, Tsirigos A, Welstead GG, Trimarchi T, Bakogianni S, Xu L, et al. Contrasting roles of histone 3 lysine 27 demethylases in acute lymphoblastic leukaemia. Nature. 2014;514(7523):513-7.

\section{Submit your next manuscript to BioMed Central and we will help you at every step:}

- We accept pre-submission inquiries

- Our selector tool helps you to find the most relevant journal

- We provide round the clock customer support

- Convenient online submission

- Thorough peer review

- Inclusion in PubMed and all major indexing services

- Maximum visibility for your research

Submit your manuscript at www.biomedcentral.com/submit

) Biomed Central 